\title{
Providing a sensory basis for models of visual information acquisition
}

\author{
GEOFFREY R. LOFTUS and THOMAS A. BUSEY \\ University of Washington, Seattle, Washington \\ and \\ JOHN W. SENDERS \\ University of Toronto, Toronto, Ontario, Canada
}

\begin{abstract}
Our major goal is to account for some simple digit-recall data with a theory that integrates two models from two scientific traditions. The random-sampling model, founded in the memory and attention literature, holds that (1) stimulus features are randomly sampled throughout the course of stimulus presence and (2) proportion correct recall is equal to the ratio of sampled features to total features. The linear-filter model, founded in the vision and sensation literature, holds that the initial stages of the visual system act as a low-pass temporal filter on the input stimulus, resulting in a time-varying sensory response in the nervous system. We report two experiments in which a variable-duration, masked, four-digit string had to be immediately recalled. Experiment 1 was designed principally to replicate past data confirming the basic randomsampling model. Like others, we were able to confirm the model only by endowing it with an additional processing-delay assumption: that feature sampling does not begin until the stimulus has been physically present for some minimal duration. Experiment 2 was an extension of Experiment 1 in which the target stimulus was preceded, $250 \mathrm{msec}$ prior to its onset, by a $50-\mathrm{msec}$ pre-presentation of the same stimulus called a prime. The Experiment 2 results allowed the following conclusions. First, the initial processing delay found in Experiment 1 is immutably tied to stimulus onset; that is, if there are two stimulus onsets, separated even briefly in time, there are two associated processing delays. Second, processing rate is essentially unaffected by the prime's presentation. Third, being presented with a 50-msec prime is equivalent, in terms of memory performance, to increasing unprimed stimulus duration by approximately $30 \mathrm{msec}$; the prime can thus said to be worth $30 \mathrm{msec}$ of additional exposure duration. This third conclusion seems superficially paradoxical, in the sense that one would expect that having seen a 50-msec prime would be equivalent to increasing exposure duration by at least the same $50 \mathrm{msec}$. However, both the initial processing delays and the 30 -msec prime's worth are natural consequences of our theory that conjoins the random-sampling model with the linear-filter model.
\end{abstract}

Two fundamental questions in perception are, What is the nature of information acquisition that follows the onset of a stimulus, and how is such information acquisition influenced by other priming stimuli presented prior to the stimulus? In this article, we report two experiments that bear on both of these questions as they apply to a digitrecall task in which a four-digit string is presented for varying durations to an observer who must immediately recall as many of the digits as possible.

Such tasks have often been accounted for by one form or another of a random-sampling model. Variants of random-sampling models have long enjoyed popularity, both in the learning literature (e.g., Neimark \& Estes, 1967) and in the attention and perception literature (e.g.,

This research was supported by an NIMH grant to G.L. and an NIMH predoctoral fellowship to T.B. We thank Claus Bundesen, Charles Eriksen, Jim Townsend, and an anonymous reviewer for very helpful comments on an earlier version of the manuscript. Correspondence should be addressed to G. R. Loftus, Department of Psychology, University of Washington, Seattle, WA 98195 (e-mail: gloftus@u.washington.edu).
Kowler \& Sperling, 1980; Loftus \& Kallman, 1979; Massaro, 1970; Rumelhart, 1970; Shibuya \& Bundesen 1988; Townsend, 1981). By what we will refer to as the basic random-sampling model, a stimulus is composed of a set of relatively homogeneous features. An observer randomly samples and acquires these features, both while the stimulus is physically present and, if the stimulus is unmasked, for a brief time following stimulus offset. The probability of a correct response is equal to the proportion of acquired features.

In investigations of attention, the basic random-sampling model has been used as a tool to address various questions such as the following: How is attention distributed over the array items? Is processing capacity limited or unlimited? Does information acquisition occur serially or in parallel? To what degree are there interitem dependencies? How are to-be-reported targets in the display distinguished from not-to-be-reported distractors? Different random-sampling models make different additional assumptions (e.g., assumptions about attention distribution over different members of the array) to address these questions. 
In contrast to the profluence and precision of such attentional assumptions, there has been a relative paucity of assumptions about the nature of the sensory processes that underlie the presumed sampling. Either implicit or explicit in the various sampling models are the assumptions that (1) there is some unidimensional sensory representation of the stimulus that materializes full-blown either at the instant of stimulus onset or shortly thereafter; (2) that in the absence of a mask, the representation decays, eventually to zero, with decay beginning at stimulus offset; and (3) that the magnitude of this sensory representation is constant during stimulus presence and independent of stimulus duration. Although this viewpoint is simple and mathematically convenient, it is at odds with a great deal of sensory literature which implies that (1) the sensory representation rises gradually, not abruptly, following stimulus onset; (2) the peak strength of the representation-and hence the onset of decay-occurs sometime after stimulus offset; and (3) the sensory representation's peak magnitude is positively related to stimulus duration (e.g., de Lange, 1952; Ives, 1922; Kelly, 1961; see Cornsweet, 1971, pp. 384-418, and Watson, 1986, for thorough discussions of this literature).

Our principal goal in this article is to supplement the basic random-sampling model by conjoining it with a linear-filter model, developed primarily in the vision literature, which generates the kind of sensory representation that we have just described. The supplemented randomsampling model that results from this marriage can account for several phenomena that are not predicted by any kind of random-sampling model in isolation.

The remainder of this article will be organized as follows. After describing our experimental task and the rationale for using it, we will detail the logic and the predictions of the basic random-sampling model. We will then present some data from the literature, along with several new experiments performed with a digit-recall paradigm, and we will report two phenomena that are not predicted by the random-sampling model. Finally, we will describe how the linear-filter model can be gracefully conjoined with the random-sampling model and show how these previously unpredicted phenomena come about as natural consequences of this conjunction.

To forestall confusion, we must emphasize that, throughout this article, we will be considering two randomsampling models. The basic model, which has appeared frequently in the literature, makes quite specific predictions about our digit-recall task; in particular, it implies a linear reiation between stimulus duration and our performance measure. The supplemented model results from conjoining the linear-filter model with the basic randomsampling model. The supplemented model makes predictions that are similar, but not identical to, those of the basic model. When we present data graphically, the predictions of both models will be provided; predictions of the basic model will be indicated by dashed lines, and those of the supplemented model, by solid lines.

\section{Digit-Recall Task}

Because we were primarily interested in investigating the relation between sensory and cognitive processes, we sought a task complex enough so that the fundamental perceptual/cognitive processes of attention, pattern recognition, and transfer of information to short-term storage would be required for it to be carried out. At the same time, however, we wanted the task to be simple enough so that the basic random-sampling model would not need to be burdened with extraneous assumptions in order to account for task performance.

A task that meets these requirements is as follows. A four-digit string is presented to an observer who must then immediately report as many of the digits as possible, in their correct positions. The stimulus' exposure duration is varied, and the stimulus is always followed immediately by a noise mask. The mask's luminance is such that it completely obliterates all stimulus features during simultaneous stimulus-and-mask presentation. We assumed that a mask immediately halted perceptual processing (cf. Eriksen, 1980; Sperling, 1964b). ${ }^{1}$

This task fulfills the desiderata listed above. Unlike, say, a simple threshold-detection task, digit recall is complex enough to require the fundamental processes enumerated above. At the same time, the task involves a stimulus that should not exceed short-term memory capacity and thus does not require assumptions about subjects' strategies about what to transfer to short-term memory (see, e.g., Rundus, 1971); involves a stimulus that is immediately masked, so that it does not require assumptions about iconic decay (see, e.g., Averbach \& Sperling, 1961); involves items of relatively homogeneous difficulty, so that it does not require corrections for averaging artifacts (see, e.g., Kowler \& Sperling, 1980); and does not involve partial report, so that it does not require assumptions about probe detection and utilization (see, e.g., Sperling, 1960).

\section{Predictions of the Basic Random-Sampling Model}

Data in this digit-recall paradigm can be conveniently presented in the form of a performance curve that relates the proportion of digits recalled to the stimulus duration. To derive the basic random-sampling model's predictions for the performance curve's shape, we reason as follows. Stimulus features are assumed to be randomly sampled with replacement at a rate (expressed as proportion total features/millisecond) that is invariant throughout the stimulus' physical presence. ${ }^{2}$ We refer to this invariant rate as the raw feature-sampling rate. A sampled feature is always acquired; that is, it is stored in short-term memory, where it subsequently forms part of the basis for stimulus recall. The nature of a random-sampling process dictates that new features-those that have not been sampled previously - are sampled at a lower rate; the more the features that are acquired and the fewer the new features that are left in the stimulus, the lower the rate becomes. More 
precisely, the rate of acquiring new features is proportional to the proportion of new features remaining in the stimulus. If $I(t)$ is the proportion of already acquired stimulus features at time $t$ following stimulus onset, then 1.0 $-I(t)$ is the proportion of new features remaining to be acquired, and $r(t)$, the rate of new-feature acquisition, must be

$$
r(t)=\frac{1.0-I(t)}{c},
$$

where $c$ is a constant that has units of time (milliseconds, in the present treatment). This logic is summarized in Appendix $\mathrm{A}$, which shows that if the stimulus remains present for a duration of $d \mathrm{msec}$, then the proportion of acquired features, $I(d)$, is

$$
I(d)=1.0-e^{-d / c} .
$$

Finally, assuming that proportion correct, ${ }^{3} p$, is equal to proportion acquired features, Equation 2 becomes

$$
p=1.0-e^{-d / c},
$$

which indicates that, in terms of $p$, the model predicts performance curves to be exponential.

\section{Interpretation of $1 / \mathrm{c}$ as Sampling Rate}

As noted in Appendix A, the interpretation of the parameter $c$ in Equations 1-3 is that $c$ 's reciprocal is the raw sampling rate measured in proportion total features per millisecond. So, for instance, a typical value of $c$ is $100 \mathrm{msec}$. This value would be interpreted to mean that features are sampled at a constant rate of $1 / 100=0.01$, or $1 \% / \mathrm{msec}$. It is important to reemphasize here that this is the raw sampling rate, which is assumed to remain constant throughout stimulus presence. Sampling of new features declines over time, as indicated in Equation 1.

\section{Asymptotic Performance}

At this point, we introduce what, for the model, is an annoyance. Part of our digit-recall paradigm's presumed simplicity is that a four-digit stimulus is within short-term memory capacity. Accordingly, we assumed that observers were always capable of reporting all four digitsthat is, of being perfect in the task-given sufficient stimulus duration. Given this assumption, performance curves should, in principle, asymptote at a value of 1.0 , as they did for all observers in experiments reported by Loftus, Duncan, and Gehrig (1992), who used the same paradigm. To check the validity of this assumption, we collected some control data in which observers were allowed unlimited time to see the stimuli. Performance under such circumstances was essentially perfect. ${ }^{4}$

In contrast to their behavior in this control procedure, however, observers in the actual experiments were apparently not as vigilant as they might have been: they made occasional keypress errors, attention lapses, and the like (which we call careless errors, to be distinguished from errors based on lack of stimulus information). Because of these errors, the actual asymptotic value was sometimes less than 1.0. So the model is forced to take this into account. If we assume that such careless errors are independent of stimulus duration, Equation 3 must be modified to become

$$
p=A\left(1.0-e^{-d / c}\right),
$$

where $A$ is the asymptotic value-that is, 1.0 minus the proportion of careless-error trials. We can rewrite the performance curve prediction as

$$
\frac{p}{A}=1.0-e^{-d / c} .
$$

Figure 1 illustrates the shape of performance curves generated by Equation 5 . This figure actually embodies two separate predictions: first, there should be some asymptotic value, $A$, such that $p / A$ is exponentially related to duration, $d$, and second, the curve should pass through the origin (i.e., no matter how small $d$ is, $p / A$, and thus $p$, should be greater than zero).

\section{Transformation to Linear Performance Curves}

For convenience, we define a new performance measure, $P$, as follows:

$$
P=-\ln \left(1.0-\frac{p}{A}\right)
$$

Writing Equation 5 in terms of $P$ rather than $p$ implies that

$$
P=\left(\frac{1}{c}\right) d
$$

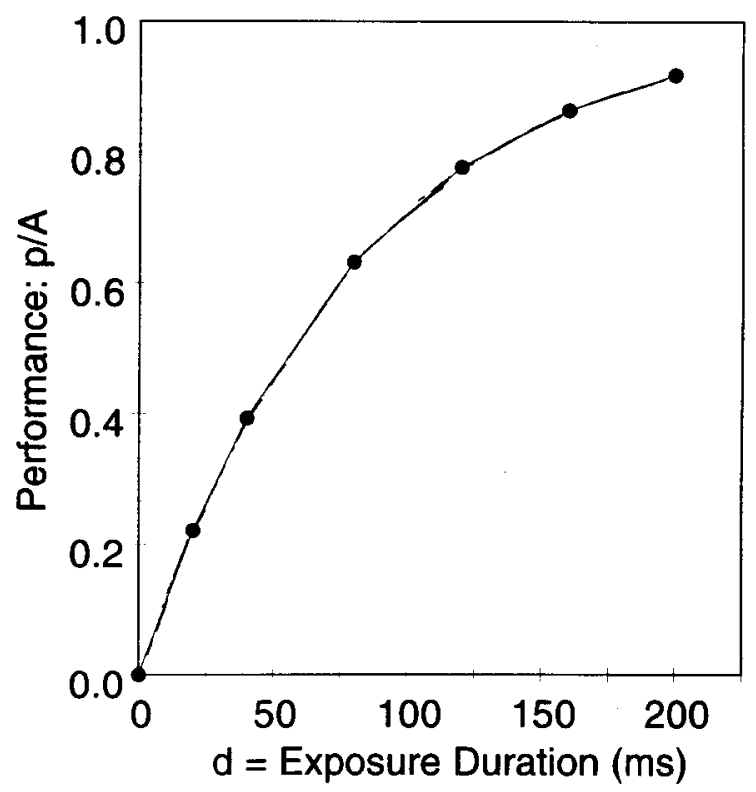

Figure 1. An example of an exponential performance curve. Because performance, $p$, is corrected for asymptotic value, the curve asymptotes at 1.0 . 
That is, $P$ is predicted by the basic random-sampling model to be a linear function of $d$ with a slope of $1 / c$ and a zero intercept. Note that $P$ is conceptualized as the logarithm of proportion error's reciprocal: accordingly, larger $P$ values, like larger $p$ values, correspond to better performance.

\section{On the Limits of the Basic Model}

The basic random-sampling model is quite simple. It makes concomitantly simple predictions. The model is sufficiently rooted in the literature, and it accounts sufficiently well for a variety of extant data that it is useful to begin exploring where it fails. In the sections that follow, we will show that the basic model indeed fails in two related ways that are entirely consistent with the frontend sensory model that we will eventually describe.

The first way in which the model fails-demonstrated by previous data, and by our Experiment 1 data-is that the performance curve, while linear, does not pass through the origin as dictated by Equation 6 . Instead, it intersects the duration axis at values ranging from 13 to $102 \mathrm{msec}$.

The second way in which the basic model fails is more subtle. In our Experiment 2, we use what we refer to as a priming technique, in which the variable-duration stimulus is in some conditions preceded, $250 \mathrm{msec}$ prior to its onset, by a 50-msec "pre-presentation" of the exact same stimulus called a prime. It will turn out that an apparent paradox emerges from a comparison of performance for such primed stimuli with performance for their normal, or unprimed counterparts. In particular, a $d$-msec primed stimulus leads to the same performance level as does a $(d+30)$-msec unprimed stimulus. We will demonstrate that this is inconsistent with a general prediction of the basic model, which is that if a $d$-msec primed stimulus leads to the same performance as a $(d+x)$-msec unprimed stimulus, then $x$ must be at least the physical duration of the prime, which, as noted, was $50 \mathrm{msec}$.

\section{Shibuya and Bundesen's Data: The "Processing Delay"}

The prediction embodied in Equation 6-that performance, $P$, is proportional to stimulus duration, $d-$ can be evaluated by using previously published data. Shibuya and Bundesen (1988) reported an experiment in which an array of mixed digits and letters were displayed. The observer's task was to report the digits only, ignoring the letters. Exposure duration varied from 10 to $200 \mathrm{msec}$, and the displays were followed by a mask at offset. The displays differed both in the number of digits and in the number of letters.

In two of the conditions, there were no letters, and the number of digits was either 2 or 4 . These two conditions provide the simple task we seek; accordingly we use them as an initial test of Equation 6. ${ }^{5}$ For each condition of each of Shibuya and Bundesen's (1988) 2 observers, we determined the asymptote value $(A)$ that maximized the Pearson $r^{2}$ value between $d$ and $P$. In carrying out this linear regression, we allowed both the slope and the intercept to vary; thus, Equation 6 predicts that expectation of the intercept is zero. The resulting fits are shown in Figure 2; here the symbols represent the data points, the dashed lines represent the best linear-regression fits, and the solid lines
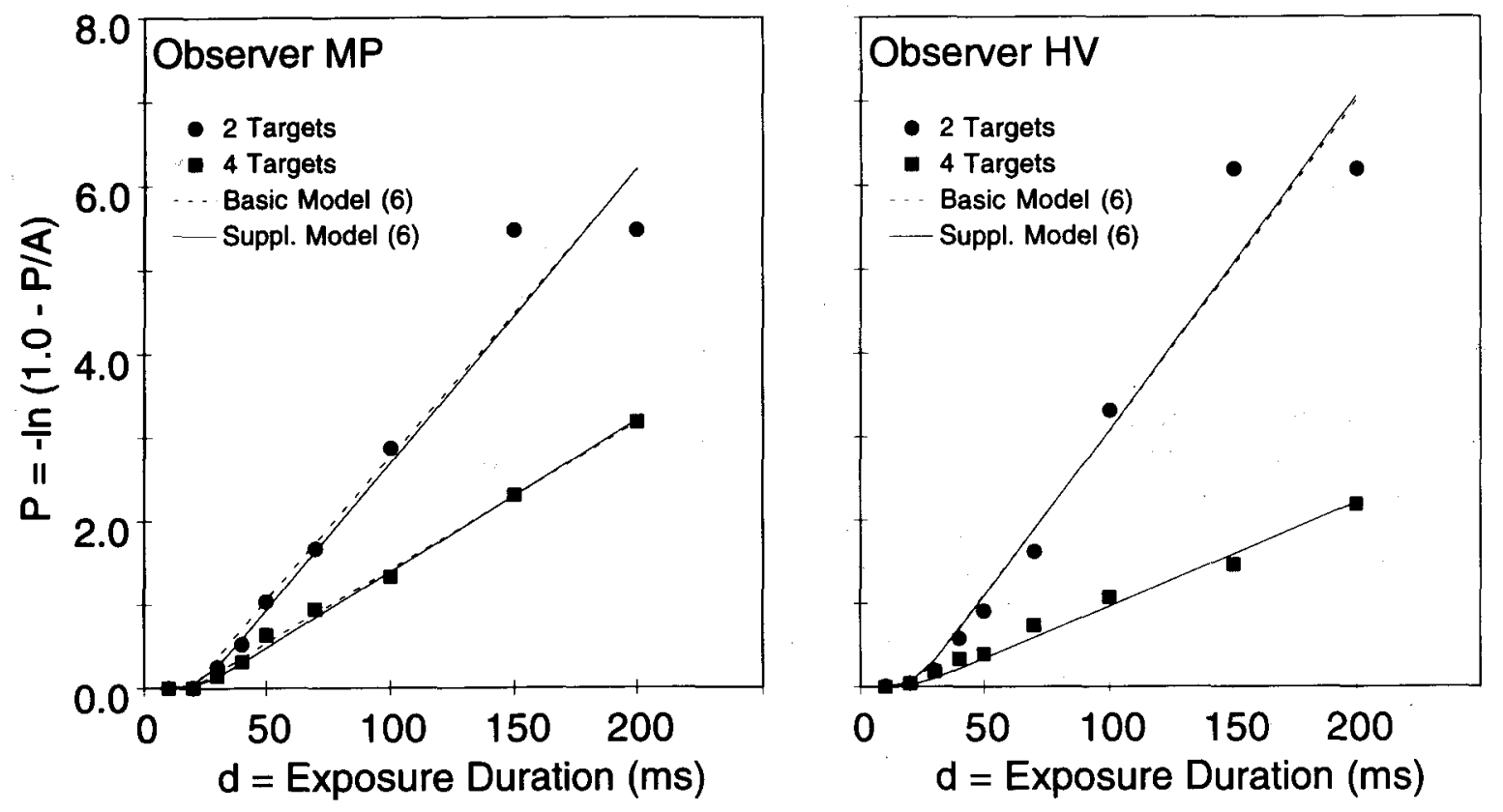

Figure 2. Performance curves from two of Shibuya and Bundesen's (1988) conditions. Different panels represent different observers. Dashed lines represent best basic-model (linear regression) fits; solid lines represent best supplemented-model fits. Parenthesized numbers in each panel's legend refer to the number of parameters required to fit each model to all data points in the panel. 
represent the best fits of the to-be-described supplemented model. The parenthesized numbers in the legend after "Basic Model"' (6) and "Suppl. Model" (6) refer to the number of parameters required to fit each model. Thus, six parameters were required for the basic model's linearregression fit to the data within each panel: an asymptote, a slope, and an intercept for each curve. (As it happens, six parameters were also required for the supplemented model; these will be discussed later in this article.)

Table 1 provides the best-fitting regression parametersthe inverse slope, $c$; the duration-axis intercept, $L$; and the asymptote, $A$-along with the Pearson $r^{2}$ s. Several results are immediately obvious.

\section{Linearity}

First, in accord with Equation 6, the curves for $T=4$ are highly linear ( $r^{2}>.99$ for both observers). Second, the curves for $T=2$ are similarly linear up to an exposure duration of about $150 \mathrm{msec}$ and then level off. We suggest that there is a possible ceiling effect for the 200 -msec data; in particular, for both the 150 -msec and the 200 -msec conditions, proportion correct $(p)$ for $T=2$ is .950 for M.P. and .975 for H.V. In principle, Equation 6 should be impervious to ceiling effects: as $d$ increases and $p$ becomes progressively closer to $1.0, P$ simply continues to rise. However, there are technical reasons for expecting ceilingeffect difficulties. For example, a single incorrect keypress, leading to a $1 \%$ reduction in $p$, would make little difference in $P$ if the reduction were, say, from $50 \%$ to $49 \%$ (in fact, a difference of .020) but would make an enormous difference if the reduction were from $98 \%$ to $97 \%$ (a difference of .405). In other words, unless the closeto-ceiling probabilities are based on a very large number of data points, relatively rare errors would lead to the kind of leveling off that is observed. In short, we believe that linearity adequately describes the Figure 2 curves.

\section{Slope Differences}

We note for the record that the performance curves are considerably shallower (i.e., $c$ is greater) for the $T=4$ than the $T=2$ condition. Shibuya and Bundesen (1988) discuss this and other between-condition differences extensively: the relations among the different number-oftarget and number-of-distractor conditions was of principal concern to them in the attentional models that they were evaluating. However, these differences are not of direct concern to us.

\section{Above-Zero Intercepts}

Finally, and of considerable interest to us, is that the curves do not pass through the origin. Instead, they intersect the duration axis at values ranging from 13 to $21 \mathrm{msec}$. This means that the basic model embodied in Equation 6 is incorrect: in order to adequately describe the data, Equation 6 must be modified to include this intercept. In particular, if $L$ (a mnemonic for liftoff) is the point at which a curve intercepts the duration axis, then the correct descriptive equation is
Table 1

Summary Statistics for Two Conditions Reported by Shibuya and Bundesen (1988)

\begin{tabular}{cccccc}
\hline Observer & Condition & $c$ & $L$ & $A$ & $r^{2}$ \\
\hline M.P. & $T=2$ & 29 & 19 & .954 & .955 \\
M.P. & $T=4$ & 57 & 19 & .865 & .997 \\
H.V. & $T=2$ & 25 & 21 & .977 & .952 \\
H.V. & $T=4$ & 87 & 13 & .873 & .992 \\
\hline
\end{tabular}

$$
P=\begin{array}{ll}
0 & \text { for } d<L \\
(1 / c) d-(L / c) & \text { for } d \geq L
\end{array}
$$

We are not the first to point out the existence of this above-zero intercept (hereafter, for ease of reference, above-zero liftoff). Rumelhart (1970) noted that "there is probably some rise time or latency associated with the onset of processing following the onset of the display" (p. 193). (Rumelhart did not, however, formally include such above-zero liftoff time in his model.) Both Shibuya and Bundesen (1988) and Townsend (1981) explicitly allowed for above-zero liftoff time in their models by including it as a parameter in their exponential equations.

We wish to emphasize that in previous models, this above-zero liftoff time has been incorporated as an ad hoc assumption whose only purpose was to account for the data; that is, its existence has not derived from more fundamental principles. Within the context of the supplemented random-sampling model described later in this article, however, an above-zero liftoff does derive from more fundamental principles.

\section{The Experiments}

In the present paper, we report two experiments. In Experiment 1 , we replicated a subset of the basic digit-recall data reported by Shibuya and Bundesen (1988) and performed some additional analyses on it. In Experiment 2, we introduced a modification of the digit-recall task in which the stimulus was presented twice on a trial, with a separation of $250 \mathrm{msec}$ of blank time.

\section{EXPERIMENT 1}

The Shibuya and Bundesen (1988) data shown in Figure 2 provide reasonable support for Equation 7's validity. However, these data came from a relatively small sample of observers, and from two conditions embedded in a much larger experiment. Experiment 1 was designed in part to replicate the Figure 2 data, and to verify the Equation 7 prediction in a purer, simpler context.

\section{Method}

The basic procedure was simple: four-digit arrays were presented for varying durations and followed by a noise mask. The observers' task was to report as many of the four digits as possible in their correct positions.

\section{Observers}

Four observers participated in Experiment 1: the first author (T.B.), a female graduate student (E.F.), a female undergraduate (S.S.), and a male undergraduate (S.T.). All observers except S.T. 
were highly practiced, having participated in a minimum of 2,000 trials prior to beginning this experiment. Observer S.T. was entirely unpracticed.

\section{Stimuli and Apparatus}

The observers viewed stimuli and a random-noise mask, all prepared as $35-\mathrm{mm}$ slides. Each stimulus slide consisted of a 4 (columns) $\times 3$ (rows) array of black digits on a white background. On each experimental trial, one digit row of one slide served as the to-be-remembered stimulus. Eighty slides were prepared and used repeatedly. The $4 \times 3 \times 80=960$ digits were selected randomly, with the restrictions that (1) each digit appear 96 times over the 960 total digits and (2) no digit appear more than twice in a given row of a slide. Each digit subtended a visual angle of $0.68^{\circ}$ vertically and $0.35^{\circ}$ horizontally. Digits were separated by $0.27^{\circ}$ vertically and $0.76^{\circ}$ horizontally.

On a given experimental trial, either the top or the bottom fourdigit row of one stimulus was the to-be-reported target. Target row was blocked over trials; accordingly, an observer always knew far in advance which row was the target.

The noise mask consisted of black visual noise on a white background.

Stimulus contrast was substantially reduced, to avoid ceiling performance. Contrast reduction was accomplished by superimposing a uniform adapting field over the stimuli and reducing stimulus luminance with the use of neutral-density filters (note that contrast varied by this procedure because the stimulus luminance changed while adapting-field luminance did not). Stimulus contrast varied from observer to observer. A summary of luminances and contrasts for both Experiment 1 and Experiment 2 is provided in Table 2.

All stimuli were displayed via Kodak projectors equipped with Gerbrands tachistoscopic shutters. A random-access projector was used to display the stimuli, while standard Carousel projectors were used to present the noise mask and a contrast-reducing uniform light field. Responses were made on a numeric keypad. All display equipment was enclosed in a soundproof box. All display and response collection was under the control of an AT-compatible computer system described by Stoddard and Loftus (1988).

\section{Design and Procedure}

There were eight stimulus exposure durations. For all observers except S.T., the unpracticed observer, duration ranged from 40 to $200 \mathrm{msec}$ in logarithmically equal steps. For S.T., the durations ranged from 20 to $300 \mathrm{msec}$ in logarithmically equal steps.

For each observer, the experiment was arranged in blocks of 80 trials. Recall that the stimuli were prepared as three four-digit rows. On any given block, only one row (top or bottom) was the to-bereported target.

Events during an 80-trial block. The sequence of events for a given 80 -trial block was as follows. First, a high or low tone (2000 or $500 \mathrm{~Hz}$ ) signaled the observer that the top or bottom row would

Table 2

Luminances (Candles/Meter ${ }^{2}$ ) and Contrasts

\begin{tabular}{lccc}
\hline & \multicolumn{2}{c}{ Luminance } & \\
\cline { 2 - 3 } & Background & Foreground & Contrast \\
\hline Adapting field & 16.58 & & \\
Fixation point & 31.07 & 17.00 & .293 \\
Mask & 67.17 & 2.35 & .933 \\
\multicolumn{4}{c}{ Experiment 1 Stimuli } \\
Observers: \\
T.B., E.F., S.S. & 19.73 & 18.47 & \\
S.T. & 28.75 & 20.36 & .033 \\
& Experiment & 2 Stimuli & \\
All observers & 19.73 & 18.47 & .033 \\
\hline
\end{tabular}

be the target row for that block (i.e., for the next 80 trials). Next, eight practice trials were presented. The exposure durations for these practice trials were selected randomly, and without replacement. Next, 72 experimental trials were presented. The eight durations were randomly intermingled over the 72 trials, with the restriction that each duration occur exactly three times within each 24-trial sequence. Stimulus-presentation order was quasirandom. ${ }^{6}$

The to-be-reported row was changed in an alternating sequence over blocks. Assignment of conditions to trials within a block was also changed over blocks.

Events during a single trial. As noted, a block consisted of 80 trials. The sequence of events within each trial was as follows. First, there was a $500-\mathrm{msec}$ tone, which warned the observer to look at a small fixation point positioned such that it would be in the middle of the upcoming stimulus (i.e., between the second and third digits of the middle row). The warning-tone frequency of either 2000 or $500 \mathrm{~Hz}$ reminded the observer which row (top or bottom) was the target during the current block. Following the warning tone, the stimulus, superimposed on the adapting field, was presented for its appropriate duration. The noise mask, which immediately followed the stimulus, was presented for $300 \mathrm{msec}$. The mask was followed by the adapting field, which remained present for the rest of the trial. The observer typed in four responses after mask disappearance, guessing on a digit if uncertain. Following responding, there was feedback in the form of four 150-msec beeps. Each beep was $2000 \mathrm{~Hz}$ if the corresponding digit had been correctly reported, and $500 \mathrm{~Hz}$ if the corresponding digit had not been correctly reported. Following feedback, there was a 300 -msec adapting-fieldonly interval prior to the start of the next trial. The adapting field was thus on constantly, except during mask presentation.

Note that there were nine instances of each duration per block. The numbers of blocks per observer were one (S.T.), four (E.F.), and six (S.S. and T.B.).

\section{Results and Discussion}

Figure 3 shows the performance curves ( $P$ as a function of $d$ ) for the 4 observers. As in Figure 2, the symbols represent the data points, the dashed lines represent the best linear fits, and the solid lines represent the best fit of the supplemented random-sampling model. Table 3 provides the best-fitting linear-regression parameter values and Pearson $r^{2}$ s for the 4 observers. The $r^{2}$ values are quite high, ranging from .979 to 1.000 for the individual subjects. This indicates that the basic random-sampling model, whose prediction is embodied in Equation 7, fits our data quite well.

\section{Liftoff Values}

The liftoff values range from 58 to $102 \mathrm{msec}$ across the 4 observers. This finding replicates the Shibuya and Bundesen (1988) data, again disconfirming the basic model embodied in Equation 6. Recall that Shibuya and Bundesen's liftoff values were smaller, ranging from 13 to $21 \mathrm{msec}$ (see Figure 1). Briefly, we believe that the difference occurs because the stimulus contrast was much lower (.033) in the present experiments than in Shibuya and Bundesen's experiments (where it was approximately .97). We will address this issue in more detail when we describe the supplemented model.

\section{Serial-Position Effects}

The Figure 3 data are collapsed across the four serial positions. There are various reasons to expect that per- 


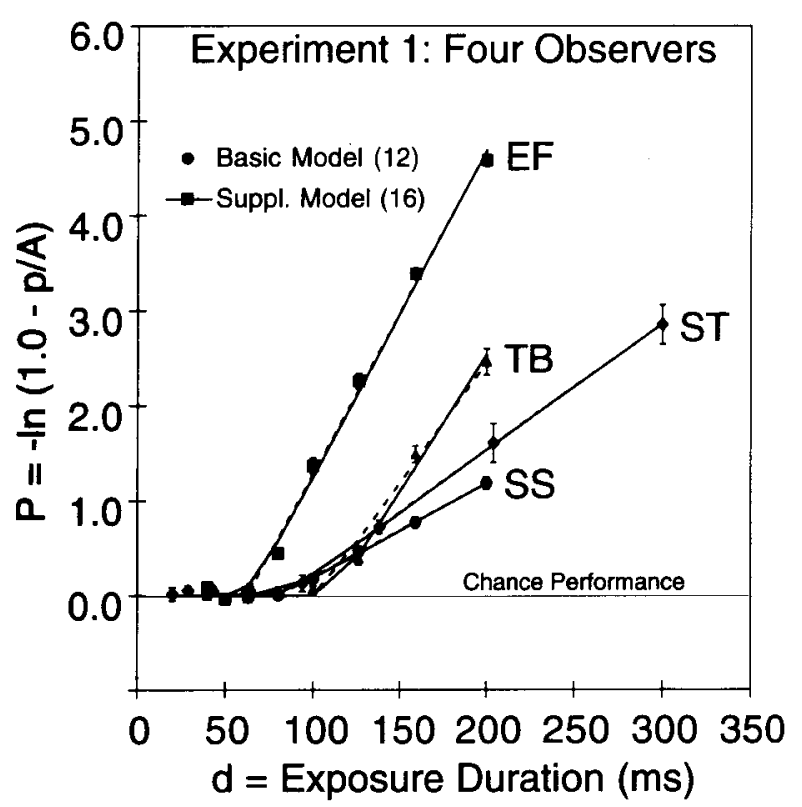

Figure 3. Experiment 1, performance curves for 4 observers. Dashed lines represent best basic-model (linear regression) fits while solid lines represent best supplemented-model fits. Error bars represent standard errors. Parenthesized numbers in the legend refer to the number of parameters required to fit each model to all data points.

Table 3

Fit of the Basic Random-Sampling Model for 4 Observers in Experiment 1

\begin{tabular}{crrrl}
\hline Observer & \multicolumn{1}{c}{$L$} & \multicolumn{1}{c}{$c$} & \multicolumn{1}{c}{$A$} & $r^{2}$ \\
\hline S.S. & 80 & 101 & .978 & .999 \\
E.F. & 58 & 35 & .992 & .979 \\
T.B. & 102 & 41 & .706 & .982 \\
S.T. & 84 & 76 & .865 & 1.000 \\
\hline
\end{tabular}

formance may vary across serial position-for example, scanning-order biases (see Townsend, 1981) or lateral masking (e.g., Wolford, 1975; Wolford \& Chambers, 1984). Figure 4 shows performance curves for the four individual serial positions (averaged over Observers E.F., S.S., and T.B., who had the same exposure durations). Again, dashed and solid lines represent best basic-model fits and best supplemented-model fits. Table 4 provides corresponding summary data: the values of $c, L$, and $r^{2}$ for the four serial positions. We estimated a common asymptotic performance for the four performance curves, which was .958.

The curves are highly linear (all $r^{2} \mathrm{~s}>.97$ ), and the liftoff durations are within $5 \mathrm{msec}$ of one another. This provides evidence that (1) the linearity of the Figure 3 data does not issue from over-serial-position-averaging artifacts, and (2) whatever the sensory or perceptual mechanism that underlies the above-zero liftoff, it operates in the same manner across serial position.

The four curves have different slopes. As noted, there are various possible reasons for these differences; distinguishing among them is not of principal concern here, however. We subsume the possible reasons under the general assertion that, for one reason or another, information is acquired from the different serial positions at different rates.

\section{EXPERIMENT 2}

In Experiment 1, we found strong support for the proposition that once it begins, information acquisition from our digit stimuli can be described well by a randomsampling model along with the extra assumption of an initial processing delay (liftoff). In Experiment 2, we ask how the putative sampling process is affected by what we call a priming stimulus, which occurs just prior to the stimulus itself. Traditional priming stimuli are related to one degree or another to the to-be-remembered stimulus. Our priming stimulus takes such relatedness to an extreme: the prime is the to-be-remembered stimulus itself.

Experiment 2 incorporated three priming conditions. A trial in the primed condition consisted of a 50-msec pre-

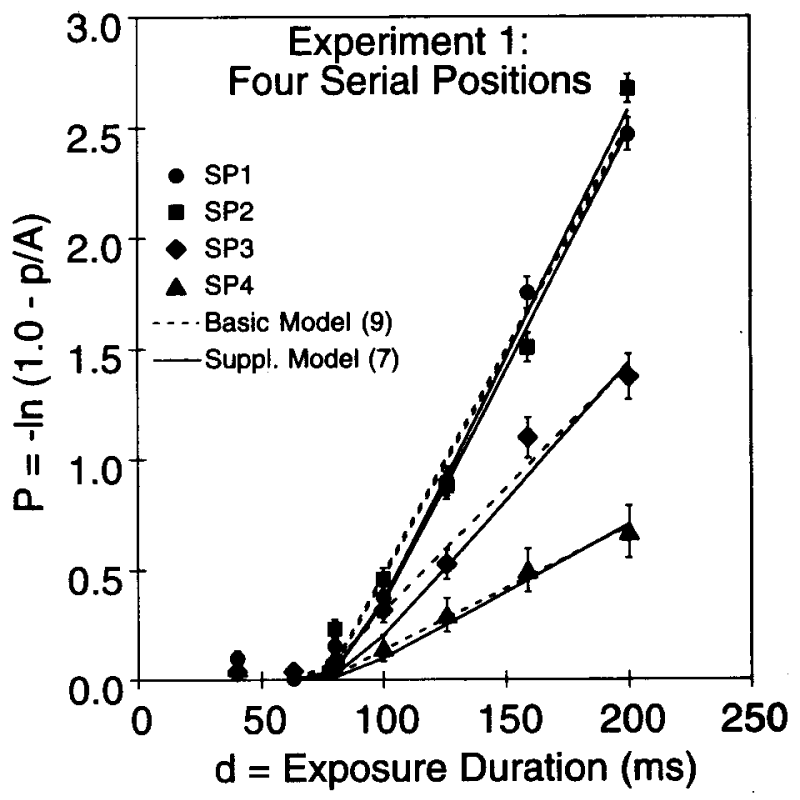

Figure 4. Experiment 1, data for four serial positions, averaged over the 3 practiced observers. Dashed lines represent best basicmodel (linear regression) fits; solid lines represent best supplementedmodel fits. Error bars represent standard errors. Parenthesized numbers in the legend refer to the number of parameters required to fit each model to all data points.

Table 4

Fit of the Basic Random-Sampling Model for Four Serial Positions in Experiment 1 (Collapsed Over 3 Observers)

\begin{tabular}{cccc}
\hline Serial Position & $L$ & $c$ & $r^{2}$ \\
\hline 1 & 77 & 49 & .991 \\
2 & 76 & 49 & .974 \\
3 & 72 & 89 & .978 \\
4 & 76 & 178 & .991 \\
\hline
\end{tabular}

Note-Common asymptote: 0.958 . 
sentation of the to-be-remembered digit string (called the prime), followed by a 250 -msec blank interval, followed by a second, variable-duration primed-stimulus presentation, followed by a mask. This primed condition was compared with two control conditions. The prime-only condition consisted of a single, unmasked, 50-msec stimulus presentation, which was followed by a 250 -msec interstimulus interval (ISI), a variable-duration additional blank time, and finally the mask. The unprimed condition consisted of a single, variable-duration, immediately masked stimulus presentation (as in Experiment 1).

\section{Possible Prime Effects}

A prime of any sort generally improves performance in almost any task. But it could do so in any of several ways. Here, we will consider four possibilities, the predictions of which are shown in Figure 5.

Each Figure 5 panel shows three curves. The unprimedstimulus performance curve is represented by the triangles, the primed-stimulus curve is represented by circles, and the prime-only curve is represented by diamonds. Note that the "duration" associated with the prime-only condition is the additional blank duration that follows the initial 250-msec delay after prime offset but prior to mask occurrence. All predicted prime-only curves are flat, reflecting our expectation, on the basis of past data, that no information would be acquired from the prime after $250 \mathrm{msec}$ following stimulus offset.

\section{Default Prediction of the Basic Model}

The random-sampling model offers a straightforward and natural prediction about the prime's effect. This is that the sampling process simply carries on following the second presentation's onset as if there had been no interruption. The primed-stimulus performance curve implied by this prediction is shown in Figure 5A. With zero duration, performance is based only on information acquired from the prime; thus, it equals prime-only performance. With additional duration, the primed curve rises.

Note that, according to the basic model, performance is equal to proportion of acquired features. This means that performance and acquired features may be discussed interchangeably. Consider the proportion of acquired features in the prime-only condition. It must logically be the
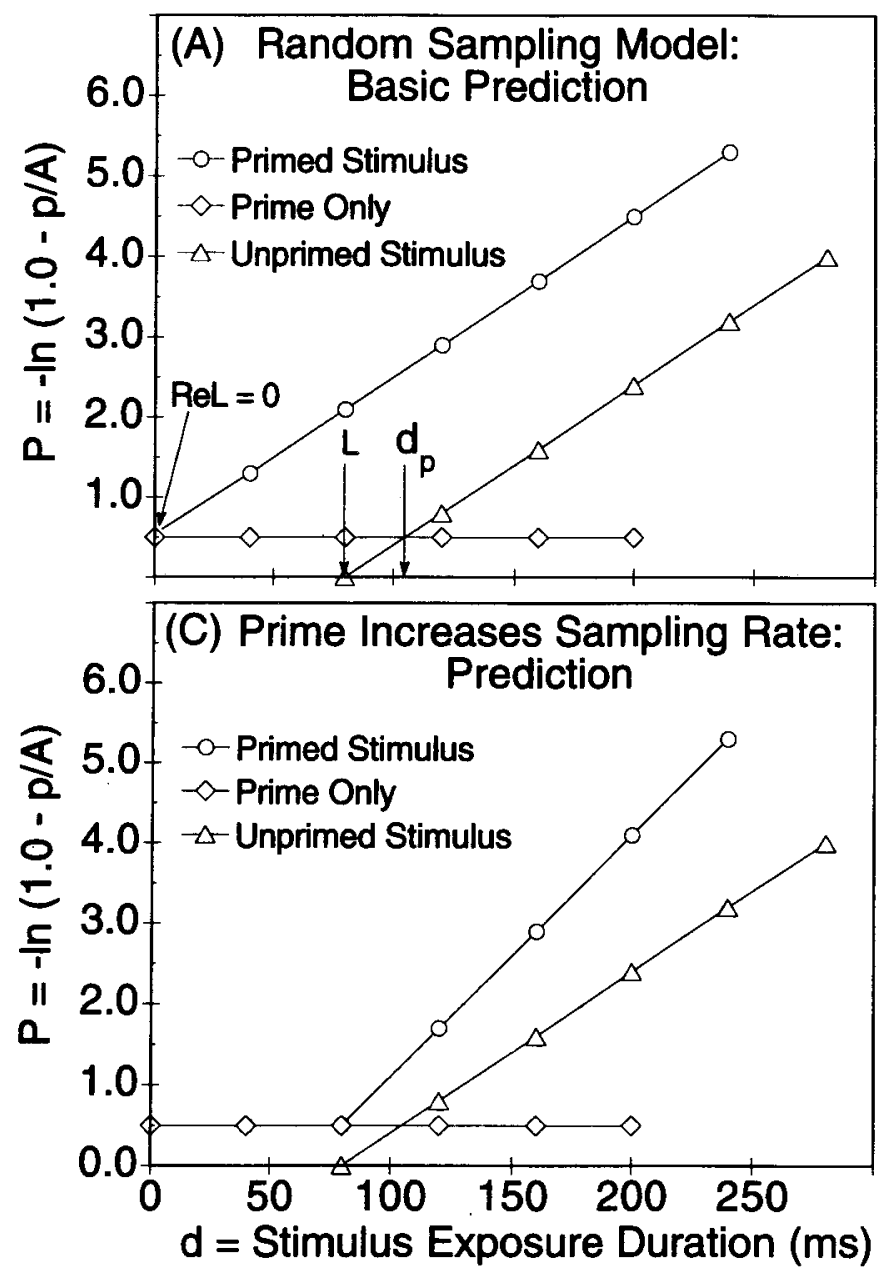
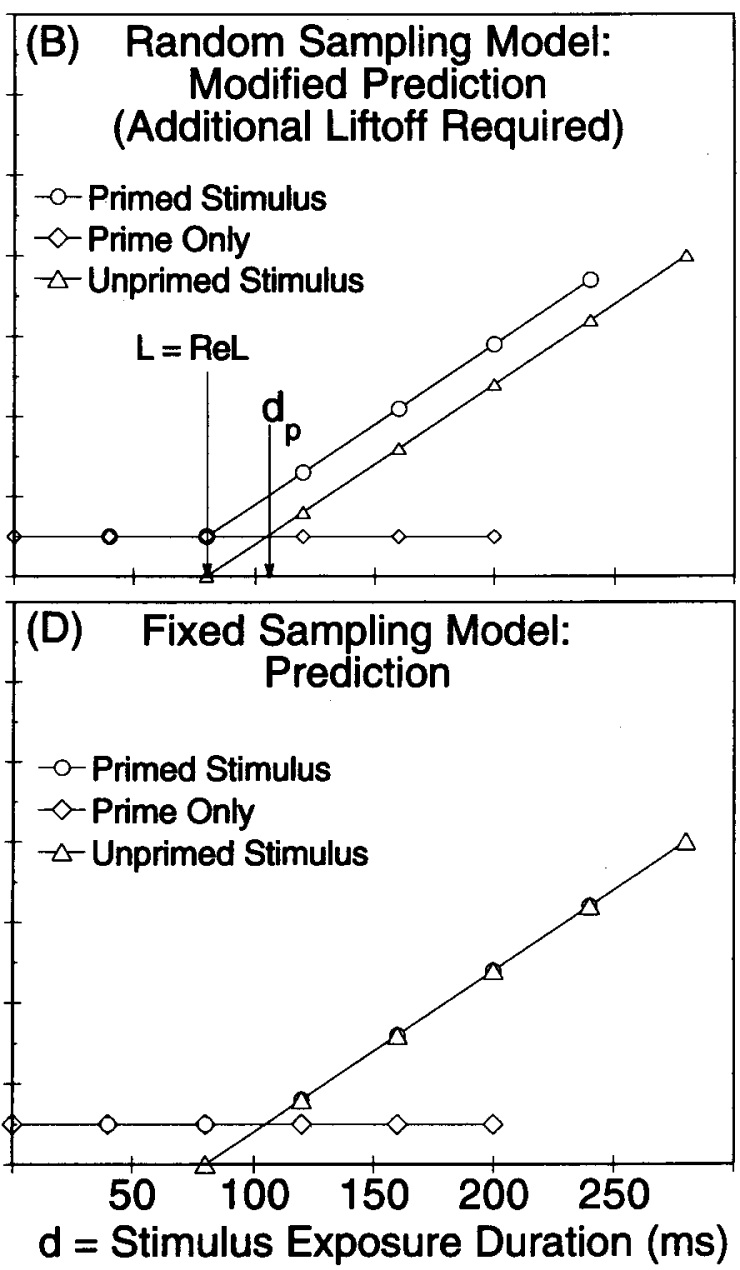

Figure 5. Performance curve predictions for Experiment 2 made by several models. 
case that the same proportion of features has been acquired at duration zero in the primed condition. Also, according to the model, the same proportion of features has been acquired following some unprimed duration, which we have labeled $d_{\mathrm{p}}$ in Figure $5 \mathrm{~A}$; note that $d_{\mathrm{p}}$ is the unprimed stimulus duration that produces performance equal to that in the prime-only condition. Because from the model's perspective the memory representation is completely determined by the proportion of acquired features, the memory representation following a $d$-msec primed presentation must be the same as the memory representation following a $\left(d+d_{\mathrm{p}}\right)$-msec unprimed presentation; accordingly, the primed and unprimed performance curves must be horizontally parallel, ${ }^{7}$ separated by $d_{\mathrm{p}} \mathrm{msec}$. (The note $\operatorname{Re} L=$ 0 at the lower left of Figure 5A is explained below.)

By this reasoning, the observed horizontal separation between the primed and unprimed curves provides a measure of the prime's effect. In particular, the experience of having just seen the prime can be said to be equivalent to, or worth, $d_{\mathrm{p}}$-msec of physical exposure duration relative to not having just seen the prime. The reasoning embodied in this assertion is reminiscent of that offered by Loftus, Johnson, and Shimamura (1985). In their experiments, visual stimuli, shown for varying durations, were followed either by an immediate mask (assumed to destroy any iconic image) or by a mask that was delayed by $300 \mathrm{msec}$ (assumed to allow an iconic image). The finding was that performance following a $d$-msec delayedmask stimulus was equal to performance followed by a $(d+w)$-msec immediate-masked stimulus for all values of $d$; thus the icon that presumably followed the delayedmask stimuli was said to be worth an additional $w$ msec of physical exposure duration. Loftus et al. have shown that one would expect this result, given the assumption that random-feature sampling proceeded in a uniform manner whether it was operating on a physical stimulus or on an iconic image. Thus, in a real sense, the icon can be viewed as equivalent to an additional physical stimulus that is added to the end of the physical stimulus. Analogously, by the reasoning that we have just described, the prime can be viewed as equivalent to a physical duration added to the beginning of the physical stimulus.

We acknowledge that on its face this last statement seems logically indisputable: the prime is a physical stimulus added to the beginning of the stimulus. The key here is that this is presumed to be the prime's only effect, and it is entirely plausible that such is not the case. Our next three predictions incorporate some form of such a notion.

\section{Modified Prediction of the Basic Model}

We have noted that, in order to fit the basic randomsampling model to extant data (both the data from Shibuya \& Bundesen, 1988, and the data from Experiment 1), we had to assume a processing delay of $L$ msec before information acquisition began to occur. The default prediction just described incorporates the assumption that whatever caused this Experiment 1 processing delay occurs only once-during the prime-in a primed trial, and will not occur again during the primed stimulus.

This assumption seems reasonable, in that one can easily imagine ways in which it could be correct. Suppose, for instance, that the observers generally fail to look at the fixation point prior to trial onset, and that it requires some time to fixate the correct location once the stimulus appears. This time would decrease the effective stimulus duration on a normal (unprimed) trial. It would not do so on a primed trial, however, since the presumed fixation adjustment would have occurred $300 \mathrm{msec}$ earlier at the start of the prime's presentation. Thus the $d_{\mathrm{p}}$-msec benefit enjoyed by a primed relative to an unprimed stimulus would accrue both from the prime itself and from the elimination of the $L$-msec processing delay that is no longer required.

An alternative possibility, however, is that the assumption is incorrect, and that the processing delay is caused by some basic sensory phenomenon that is inexorably triggered by stimulus onset. The prediction ensuing from this possibility is illustrated in Figure 5B. Even in the primed trial, an $L$-msec processing delay occurs before any information is acquired from the primed stimulus. Accordingly, primed performance would remain at baseline (primeonly) performance for $L$ msec before beginning to rise.

It is useful to introduce a new piece of notation here. Just as $L$ designates the liftoff duration, at which the unprimed performance curve departs from zero, $R e L$ designates the reliftoff duration at which primed performance departs from baseline prime-only performance. Both $L$ and $R e L$ are indicated in Figures 5A and 5B. It is evident that by the default prediction (Figure $5 \mathrm{~A}$ ) $\operatorname{Re} L=0<L$, whereas for the modified prediction (Figure $5 B$ ), $R e L=L$.

Above, we discussed the concept of the prime's worth, which is the additional duration that must be added to an unprimed stimulus's duration in order to raise its performance level to that of a primed stimulus. As we have noted, the prime's worth is estimated by the horizontal separation between the unprimed and primed performance curves. Given either of the two models whose predictions we have just described, the prime's worth is estimated to be $\left(d_{\mathrm{p}}-\operatorname{Re} L\right) \mathrm{msec}$.

\section{Does the Prime Affect Feature-Sampling Rate?}

We have considered two variants of the basic model. A critical commonality is the assumption that once sampling has begun, it proceeds at the same raw sampling rate $[o f(1 / \mathrm{c}) / \mathrm{msec}$, whether a stimulus has been primed or not. The equal slopes of the primed and unprimed performance curves in Figures 5A and 5B are implied by this common assumption.

This assumption could, however, be false. Reinitz, Wright, and Loftus (1989) showed subjects pictures of single objects either preceded or not preceded by the object's category name (their primed and unprimed conditions, respectively). Translating into terms of the present model, Reinitz et al. found that raw sampling rate in- 
creased in the primed relative to the unprimed condition. Such a prime effect in the present paradigm would be reflected in a performance-curve slope increase in the primed relative to the unprimed condition. This possibility is shown in Figure $5 \mathrm{C}$. $^{8}$

\section{Nonrandom Sampling}

A defining quality of random sampling is that there is no preferred feature-sampling order. A converse possibility - which would embody a failure of the basic modelis that there is a preferred sampling order.

To see what such a model would predict for the Experiment 2 results, consider an extreme possibility: that there is a fixed order in which features are sampled, and that this fixed sampling order is triggered by any stimulus onset. In this case, an observer would begin processing a primed stimulus by redundantly resampling all the features that had been sampled during the prime's presentation. This means that performance following any given duration in the primed condition would be equal to the maximum of either prime-only performance or unprimed performance at the same duration. This possibility is illustrated in Figure 5D, in which primed and unprimed curves overlap.

It is unlikely that such an extreme nonrandom sampling model is correct; for instance, it does not imply the linear performance curves that we and others have obtained. However, even a small degree of nonrandom sampling would imply that some features sampled during the prime would then be systematically resampled during the primed stimulus. This would drive the performance curve rightward from the random-sampling prediction (Figure 5B) toward the fixed-sampling prediction (Figure 5D). In other words, the prediction of any nonrandom sampling model is that $\operatorname{Re} L>L$.

\section{Method}

The methodology was very similar to that of Experiment 1 . The main change was that the single-presentation condition of Experiment 1 was compared with a new, double-presentation condition. As just described, there were 3 priming conditions and 6 durations within each priming condition, for 18 conditions in all.

\section{Observers}

Four observers participated: the first and second authors (G.L. and T.B.), and 2 female undergraduate students (S.S. from Experiment 1, and T.J.B.). All observers were highly practiced, having participated in a minimum of 1,700 trials before beginning this experiment.

\section{Stimuli and Apparatus}

Stimulus presentation was the same as in Experiment 1, except for two differences. First, during the initial warning tone for each trial, a fixation point appeared over the permanent fixation point. The fixation point consisted of a white square superimposed over the adapting field. Fixation-point luminance and contrast are provided as part of Table 1 . Second, all three rows in each stimulus array (rather than just the top and bottom as in Experiment 1) were used as targets.

\section{Design}

The three priming conditions, along with the durations associated with them, are schematized in Figure 6.

Unprimed-stimulus condition. In the unprimed-stimulus condition, the stimulus was presented alone for a duration ranging from 80 to $280 \mathrm{msec}$. The stimulus was preceded by a 300 -msec adaptingfield-only pause and was immediately followed by the mask. The unprimed stimulus condition provided a performance curve similar to that generated in Experiment 1.

Prime-only condition. In the prime-only condition, the 50-msec prime was presented alone, followed by a variable-duration adaptingfield-only pause. The pause's duration ranged from 250 to $450 \mathrm{msec}$ and was terminated by the mask. From pilot work we assumed that a 250-msec stimulus-mask ISI following stimulus offset would be sufficient for all perceptual stimulus processing to be completed and, accordingly, that prime-only performance would not vary as a function of pause duration. This prime-only condition provides a baseline performance level.

Primed-stimulus condition. In the primed-stimulus condition, the stimulus was presented for $50 \mathrm{msec}$, followed by a 250 -msec adapting-field-only pause, followed by a second presentation of the same stimulus. The second presentation ranged in duration from 40 to $240 \mathrm{msec}$ and was followed immediately by the mask. As noted earlier, we call the first stimulus presentation the prime, and the second presentation the primed stimulus.

As can be seen in Figure 6, the primed-stimulus condition is the combination of the prime-only and unprimed-stimulus conditions.

\section{Procedure}

Each observer participated in twenty-four 72-trial blocks. Within each block, the 18 stimulus conditions were randomly intermingled over the 72 trials. The sequence of events both within a block
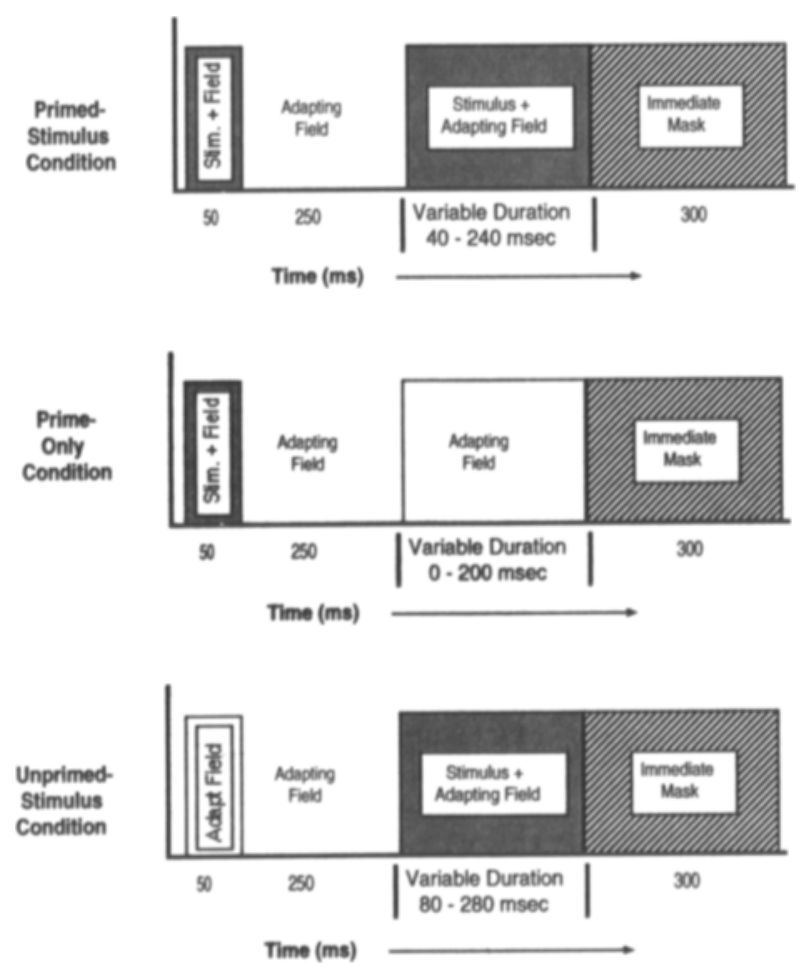

Figure 6. Conditions for Experiment 2. (See text for explanation.) 
and within a trial were the same as in Experiment 1, except for the minor changes described earlier. As in Experiment 1, the adapting field was on continuously, except during mask presentation.

\section{Results and Discussion}

Figure 7 shows the data averaged across the 4 observers. Figure 7 is organized just as each panel of Figure 5. Again, the dashed lines through the data points represent the basic model's best fit, and the solid lines represent the to-be-described supplemented model's best fit. The flat dashed line through the prime-only data points is the mean of the six prime-only duration conditions.

Table 5 provides a variety of summary statistics for both the data averaged across observers (bottom row) and individual observers' data. Table 5 is organized as follows. Columns 2-4 provide performance curve information (estimates of $c$ and $L$, plus the Pearson $r^{2}$ ) for the unprimed condition; similarly, columns 5-7 provide this information for the primed condition. (Recall that for the primed condition, $R e L$ is the reliftoff time from prime-only baseline performance.) Column 8 provides the common asymptote estimated for both conditions. Column 9 provides the mean performance in the prime-only condition. Column 10 provides the prime's worth, defined to be $\left(d_{\mathrm{p}}-\operatorname{ReL}\right)$ (see discussion above). Finally, for comparison with data reported by Loftus et al. (1985) and Loftus et al. (1992), column 11, the rightmost column, provides our estimate of the worth of the icon that followed the prime (recall that the prime was unmasked). The icon's worth was computed as $d_{\mathrm{p}}$, the duration needed by an unprimed (masked) stimulus to reach prime-only performance level, minus $50 \mathrm{msec}$, the physical duration of the prime. For instance, $\mathrm{Ob}$ server T.B. required $112 \mathrm{msec}$ in the (immediate-masked) unprimed condition to reach the (delayed-masked) primeonly performance level of 0.205 . Because the prime itself lasted $50 \mathrm{msec}$, the prime's icon was equivalent to having (i.e., was worth) an additional $112-50=62 \mathrm{msec}$ of physical exposure duration.

\section{Averaged Data}

As anticipated, the prime-only curve was flat, indicating that prime perceptual processing was completed within $250 \mathrm{msec}$ following prime offset. The Pearson $r^{2}$ values were .992 for both the primed-stimulus and the unprimedstimulus performance curves. The $c$ values were estimated to be identical $(97 \mathrm{msec})$ in the primed and unprimed con-

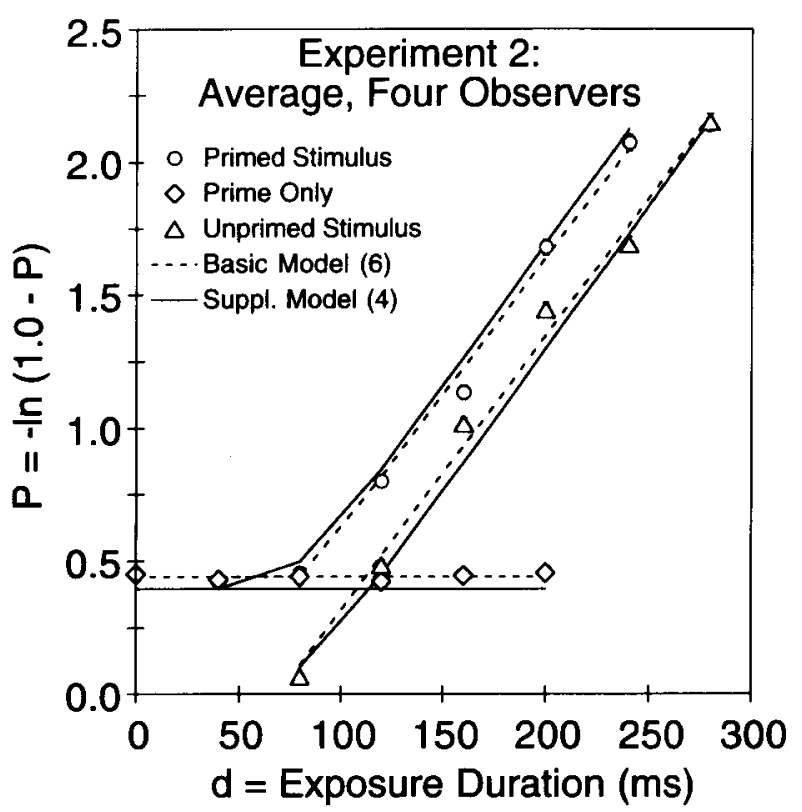

Figure 7. Experiment 2, performance curves averaged over 4 observers. Dashed lines represent best basic-model (linear regression) fits; solid lines represent best supplemented-model fits. Error bars represent standard errors. Parenthesized numbers in the legend refer to the number of parameters required to fit each model to all data points.

ditions. The primed reliftoff (84 msec) was slightly longer than the unprimed liftoff $(70 \mathrm{msec})$. Both the performance curve linearity and the equality of the primed and unprimed performance curve slopes conform well to the modified prediction of the random-sampling model (Figure 5B), although the slightly increased reliftoff relative to liftoff is consistent with (although does not of course imply) the idea that there is some systematic feature resampling during a primed presentation (cf. Figure 5D).

Icon's worth. As indicated in Table 5 (rightmost column), the mean icon's worth is estimated to be $63 \mathrm{msec}$. This is in the same general range as the corresponding values estimated by Loftus et al. (1992), which ranged from 59 to $102 \mathrm{msec}$ over 7 observers.

Prime's worth. The mean prime's worth (second column from the right) is estimated to be 30 msec: this means that a $d$-msec stimulus preceded by a $50-\mathrm{msec}$ prime produces the same performance as a $(d+30)$-msec

Table 5

Summary Data and Fit of the Basic Random-Sampling Model for 4 Observers and for the Averaged Data in Experiment 2

\begin{tabular}{|c|c|c|c|c|c|c|c|c|c|c|}
\hline \multirow[b]{2}{*}{ Observer } & \multicolumn{3}{|c|}{ Unprimed } & \multicolumn{3}{|c|}{ Primed } & \multirow{2}{*}{$\begin{array}{c}\text { Asymptote } \\
A\end{array}$} & \multirow{2}{*}{$\begin{array}{c}\text { Prime Only } \\
P\end{array}$} & \multirow{2}{*}{$\begin{array}{c}\text { Prime's } \\
\text { Worth }\end{array}$} & \multirow{2}{*}{$\begin{array}{l}\text { Icon's } \\
\text { Worth }\end{array}$} \\
\hline & $c$ & $L$ & $r^{2}$ & c & $\operatorname{ReL}$ & $r^{2}$ & & & & \\
\hline T.B. & 77 & 96 & .988 & 77 & 98 & .891 & .769 & .205 & 14 & 62 \\
\hline G.L. & 132 & 54 & .968 & 155 & 88 & .919 & .993 & .559 & 40 & 78 \\
\hline S.S. & 99 & 69 & .984 & 92 & 81 & .948 & .759 & .457 & 33 & 64 \\
\hline T.J.B. & 77 & 67 & .981 & 62 & 85 & .990 & .915 & .540 & 24 & 59 \\
\hline Mean & 97 & 70 & .992 & 97 & 84 & .992 & .857 & .440 & 30 & 63 \\
\hline
\end{tabular}

Note-Prime's worth and icon's worth are given in milliseconds. 
stimulus not preceded by a prime. At first blush, it seems odd that a prime's worth, in terms of additional physical exposure duration $(30 \mathrm{msec})$, is not even as great as the prime's actual physical duration $(50 \mathrm{msec})$. We discuss this issue at greater length below.

\section{Individual Observers}

As Table 3 indicates, individual-observer data were somewhat noisy, with $r^{2}$ values ranging from .891 to .990 . However, no observer showed consistent departures from linearity.

Primed and unprimed $c$ values. For T.B. and S.S., the estimated $c$ values were quite similar for the primed and unprimed curves. For G.L. and T.J.B., the $c$ values are somewhat different for the two curves. For T.J.B., it appears possible that the prime increases sampling rate. However, if we accept this conclusion, we are forced to similarly admit that for G.L., the prime decreases the sampling rate, contrary to any prediction. We will consider this issue at greater length in conjunction with the supplemented model that we describe below.

Liftoff and reliftoff. For all observers, the estimated primed liftoff (reliftoff) was longer than the estimated unprimed liftoff.

Icon's worth. The estimated icon's worth ranged from 59 to $78 \mathrm{msec}$ over the 4 observers. As noted, this is within the general range of past estimates.

Prime's worth. The prime's worth ranged from 14 to $40 \mathrm{msec}$ across observers.

\section{The Prime's Worth Paradox}

The mean prime's worth was $30 \mathrm{msec}$, which is less than the prime's physical duration of $50 \mathrm{msec}$. As we have just noted, the prime's worth was less than the prime's duration for all 4 observers.

This finding seems paradoxical, and it is not predicted by the basic random-sampling model. To understand these assertions, recall first that the ISI between prime offset and primed-stimulus onset was $250 \mathrm{msec}$. Suppose, for a moment, that the ISI had actually been $0 \mathrm{msec}$. Because the prime itself lasted $50 \mathrm{msec}$, a $d$-msec "primed stimulus" would, in that case, be physically identical to a $(d+50)$-msec "unprimed stimulus." This, of course, would unambiguously imply that performance following a $d$-msec primed stimulus would be identical to performance following a $(d+50)$-msec unprimed stimulus; thus, the prime would be worth $50 \mathrm{msec}$.

One might expect that with the actual ISI of $250 \mathrm{msec}$, the prime would be followed by an iconic image from which information could be acquired that would increase the prime's worth. Instead, the prime's worth shrank, to a mean of $30 \mathrm{msec}$. We will see below that this "prime's worth paradox" becomes resolved within the context of the supplemented random-sampling model.

\section{GENERAL DISCUSSION}

Let us first consider the prime's effect on processing of the primed stimulus. Recall that we offered several can- didates (see Figure 5). Clearly we can reject the hypothesis embodied in Figure 5A; reliftoff is nowhere near zero for any observer. Accordingly, we rule out the possibility that whatever the processes that are responsible for the information-processing delay (liftoff), they need only be carried out once during a brief presentation. Apparently such processes are intimately and automatically tied to stimulus onset.

The data are close to the prediction embodied in Figure $5 \mathrm{~B}$. However, there is marginal support for the models indicated in Figures 5C and 5D that, in some fashion, are contrary to a pure random-sampling model.

First, the finding that reliftoff is slightly higher than liftoff is consistent with various possibilities. One that strikes us as reasonable is that there is some slight preferred order for feature sampling, and that this order is tied to stimulus onset. Given that some features upon which performance is based are systematically acquired early in the process, they would be sampled first during the prime and again, redundantly, during the primed stimulus. Thus, the prime itself is not quite as useful as it would be if sampling were completely random. Second, Observers T.J.B. and G.L. provide some evidence that the prime can affect the sampling rate during the primedstimulus presentation.

\section{THE SUPPLEMENTED MODEL}

Two highly robust findings are not predicted by the basic model. The first is the initial processing delay reflected both in the above-zero liftoff and in the above-zero reliftoff. The second is the prime's worth paradox: a 50msec prime preceding a primed stimulus is worth less in terms of additional physical exposure than the physical duration of the prime itself. We now describe a supplement to the random-sampling model that implies both these findings.

\section{The Time Course of a Hypothetical Sensory Response}

We (and others) have informally characterized information as "acquired from the stimulus." This is not, of course, what actually happens. Rather, information is acquired on the basis of some perceptual representation that the stimulus engenders. Let us call such a representation a "sensory response." Loftus et al. (1992), Loftus and Ruthruff (in press), and Busey and Loftus (in press) have formalized the idea of a sensory response and shown how it accounts for several different phenomena, including the icon's worth, certain duration-intensity tradeoffs, and binocular combination of information. We now show how it accounts for the present data.

\section{The Linear-Filter Assumption}

The model's basic assumption is, as suggested by many past investigators, that the initial stages of the visual system act as a linear, low-pass temporal filter that operates on the physical stimulus (see, e.g., Sperling, 1964a, and Watson, 1986). The output of this filter is a sensory- 
response function, $a(t)$, that relates the magnitude of whatever neural activity represents stimulus presence to $t$, the time since stimulus onset. Examples of such functions are shown in Figure 8 for stimuli of four durations, ranging from 25 to $200 \mathrm{msec}$. The vertical lines represent stimulus offset for each of the indicated functions; for most stimuli in our experiments, a mask occurs at this time.

Detailed derivations of these functions are presented elsewhere (e.g., Loftus et al., 1992, pp. 545-547). Briefly, the equation that generates them is

$$
a(t)=\begin{array}{ll}
\phi G(t) & \text { for } t \leq d \\
\phi[G(t)-G(t-d)] & \text { for } t>d
\end{array}
$$

where $\phi$ is stimulus contrast and $G(x)$ is the integral from 0 to $x$ of the impulse-response function. ${ }^{9}$ The impulseresponse function is an Erlang function of the form

$$
g(x)=\frac{(x / \tau)^{n-1} e^{-x / \tau}}{t(n-1) !}
$$

Note that Equation 9 has two parameters: $n$ is an integer $(n \geq 1)$ and $\tau$ is a real number $(\tau>0)$.

As is evident in Figure 8, a sensory-response function is temporally blurred relative to the physical stimulus that has generated it, and it lags behind that stimulus. Also, peak magnitude increases with stimulus duration.

\section{The Sensory-Threshold Assumption}

Again, as other investigators (see Watson, 1986), we assume that information acquisition begins when the response function exceeds some sensory threshold; such a threshold is indicated by the horizontal line above the ab- scissa in Figure 8. Our key assumption that links the linear-filter model to the basic random-sampling model is that the raw sampling rate at time $t$ is proportional to the magnitude by which the sensory response exceeds threshold at time $t$. In Appendix B, we show that, given this and the random-sampling assumptions, our performance measure, $P$, is proportional to the area under the sensory-response function that lies above threshold and prior to mask onset (exemplified for the 100-msec stimulus by the cross-hatched area). We refer to this area as the performance-relevant area.

One note is in order at this point. It may appear at first glance that our supplemented model essentially entails the mere replacement of the original ad hoc assumption (the above-zero liftoff) by another ad hoc assumption (the sensory threshold). There are three answers to this complaint. First, the assumption of a sensory threshold is firmly entrenched in the sensory literature, both because it explains a variety of phenomena and because it is consistent with much physiological data. Second, as we shall see shortly, the sensory threshold accounts simultaneously for liftoff, reliftoff, and the prime's worth paradox. Finally, the threshold assumption explains other data gathered in the present paradigm, including stimulus contrast effects (Loftus \& Ruthruff, in press) and effects of presenting stimuli monoptically, binocularly, or dichoptically (Busey \& Loftus, in press).

\section{Accounting for Data}

We will begin by informally demonstrating how the supplemented model accounts for the processing delays and

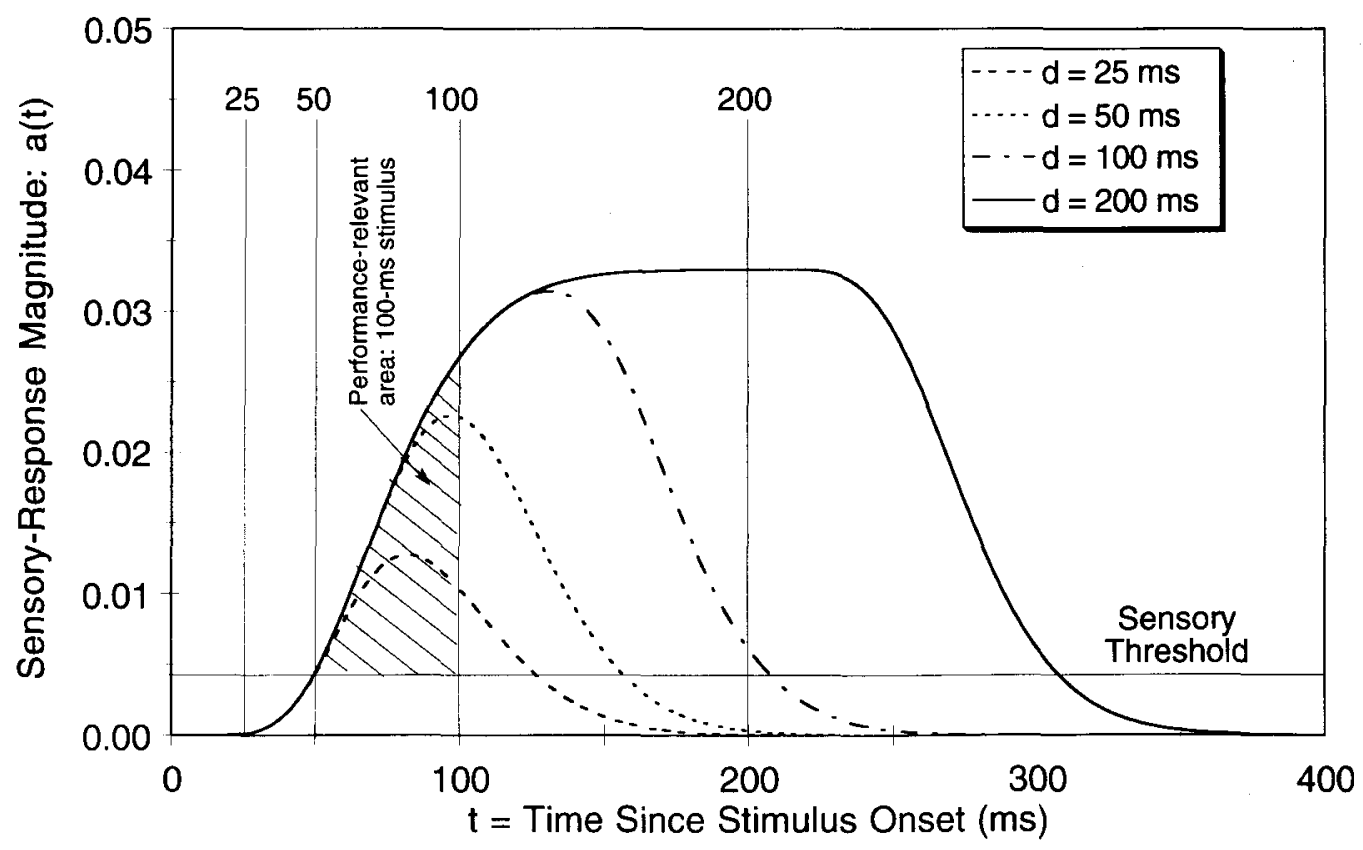

Figure 8. The linear-filter model: sensory-response magnitude as a function of time since stimulus onset, for four exposure durations. Vertical lines represent stimulus offset time. Information acquisition is assumed to occur only when sensory-response magnitude exceeds the threshold, which is indicated by the horizontal line above the abscissa. The performance-relevant area for the $100-\mathrm{msec}$ stimulus is represented by the cross-hatched area. 
the prime's worth paradox. We will then show how the quantitative predictions indicated by the solid lines in Figures $2,3,4,7$, and 11 were computed.

\section{Initial Processing Delays (Liftoff and Reliftoff)}

The model's account of the processing delay found both by Shibuya and Bundesen (1988) and in the present Experiments 1 and 2 is apparent in Figure 8. Stimuli below a certain critical duration do not exceed threshold prior to mask onset; hence, no information is acquired from them. In the illustration of Figure 8, no information would be acquired from either the $25-$ or the 50 -msec masked stimuli. ${ }^{10}$ However, any stimulus longer than $50 \mathrm{msec}$ would exceed threshold prior to mask appearance. Hence, in the Figure 8 example, the liftoff duration would be $50 \mathrm{msec}$.

It is also quite obvious why the model would predict a reliftoff for a primed stimulus. The threshold is assumed to be an immutable part of the system. Any time a stimulus is presented, it will have to be of some minimum duration in order to exceed this threshold. As indicated in Figure 9 (top panel), a delay of $250 \mathrm{msec}$ following prime offset is sufficient that the prime's sensory-response function decays essentially to zero, and the primed stimulus must start its own sensory-response function anew. Thus when the primed stimulus is sufficiently short, the performance-relevant area contributed by the primed stimulus is zero. The only performance-relevant area in such a situation would be that contributed by the prime. Accordingly, short-duration primed performance would be equal to prime-only performance, just as it actually is (see Figure 7).

\section{The Prime's Worth Paradox}

Figure 9 addresses the prime's worth paradox. Here a 100 -msec primed trial (top panel) is compared with a 130msec unprimed trial (bottom panel). In the primed trial, the prime initiates a sensory-response function that decays during the $250-\mathrm{msec}$ ISI. At time $t=300 \mathrm{msec}$, the primed stimulus appears, initiating its own sensoryresponse function, which is terminated by the mask. There are, accordingly, two performance-relevant areas, which are cross-hatched in the figure. The sum of these areas determines performance. An unprimed stimulus (bottom panel) engenders only a single sensory-response function and has one performance-relevant area. The prime's worth of $30 \mathrm{msec}$ is captured by the fact that the sum of the two performance-relevant areas in the top panel is equal to the single performance-relevant area in the bottom panel.

Threshold level determines prime's worth. As is depicted in Figure 9 (top panel), the prime's worth depends on the leftmost performance-relevant area that is contributed by the prime. The magnitude of this area is, in turn, entirely dependent on the level of the sensory threshold. If the threshold were higher (say 0.023), the prime's sensory-response function would not exceed threshold, and the prime's worth would be zero. ${ }^{11}$ The prime's worth would, in contrast, be at a maximum when the threshold
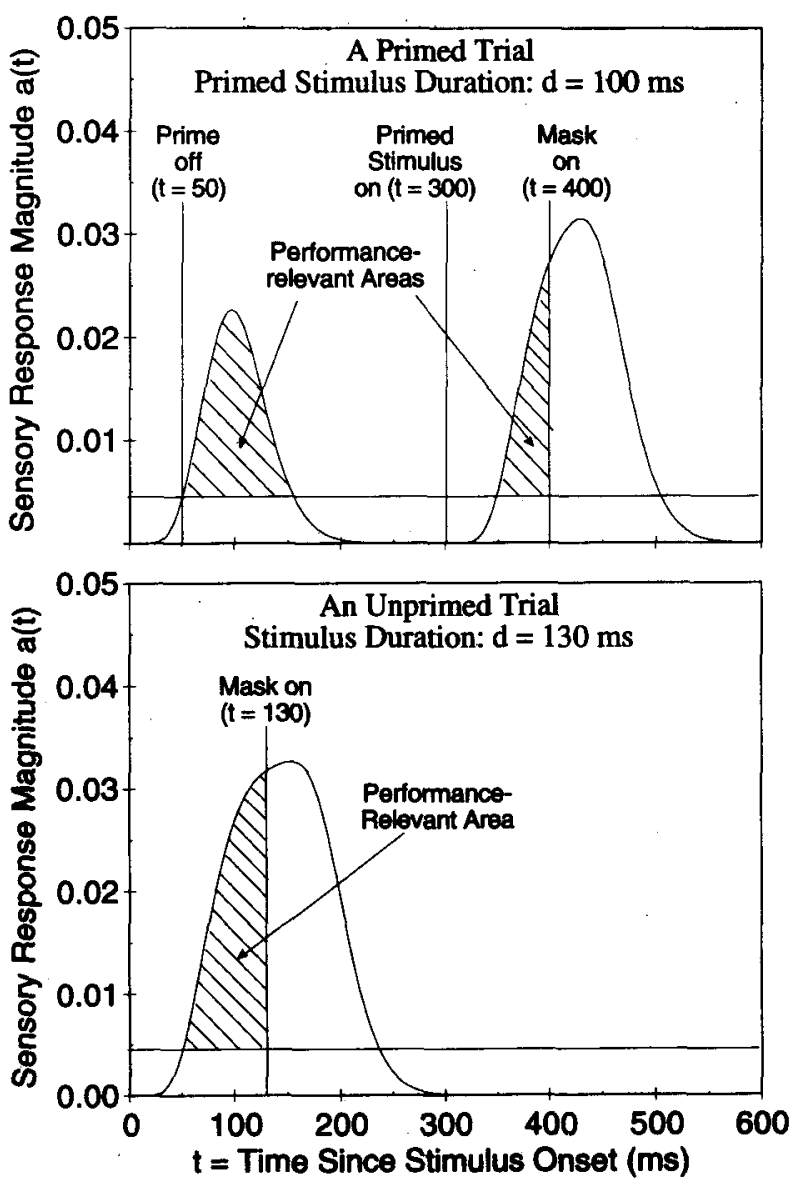

Figure 9. A sketch of how the linear-filter model accounts for the prime's worth paradox. The two panels depict the $a(t)$ functions for a primed trial (top panel) and an unprimed trial (bottom panel).

was equal to zero. ${ }^{12}$ Thus, within the context of the linearfilter model, the prime's worth paradox evaporates; depending on the threshold value, the prime's worth can easily be smaller than the prime's physical duration and, indeed, can be as small as zero.

Massed versus distributed stimulus presentation. The shrinkage of the prime's worth with an ISI of $250 \mathrm{msec}$ relative to 0 msec raises a more general issue about the effect on performance of a stimulus's physical distribution over time. Suppose a stimulus were not masked. If there were no sensory threshold, the linear-filter model would imply performance to be determined by a stimulus' total physical duration, irrespective of how the duration was distributed over time. Thus, for example, a $d$-msec stimulus would produce the same total performance-relevant area whether it was presented once for $d$ msec, or twice for $(d / 2)$ msec, separated by a blank gap. This is a fundamental consequence of linearity.

When the sensory threshold is introduced, however, a single-presentation $d$-msec stimulus gains an advantage over two separated (d/2)-msec presentations. More generally, a threshold endows a temporally massed stimulus with an advantage over a same-physical-duration, but tem- 
porally distributed, stimulus. To illustrate, consider an experiment reported by Schurman, Eriksen, and Rohrbaugh (1968, Experiment 1). The stimulus was a single to-be-reported letter that was presented as a train of 10 stimulus pulses. Pulse duration (PD) was either 2 or $4 \mathrm{msec}$, and interpulse interval (IPI) varied from 2 to $48 \mathrm{msec}$. Schurman et al. reported that performance was better for the 4- than for the 2-msec PD and that performance declined monotonically with IPI.

Figure 10 indicates how this result is accounted for by the linear-filter model, showing the sensory response function, $a(t)$, for 2- and 4-msec pulses (top and bottom panels) and for an IPI of 2 or $48 \mathrm{msec} .{ }^{13}$ For each panel, the total areas under the two $a(t)$ curves are equal. However, the above-threshold area is larger for the shorter IPI. The reason for this is that with a threshold, the peak magnitude of the $a(t)$ function becomes important: the higher the peak magnitude, the greater the degree to which $a(t)$ exceeds threshold, and the greater is the performancerelevant area.

The Schurman et al. (1968) data are shown in Figure 11 along with the fit of the supplemented model. The fit is reasonably good.
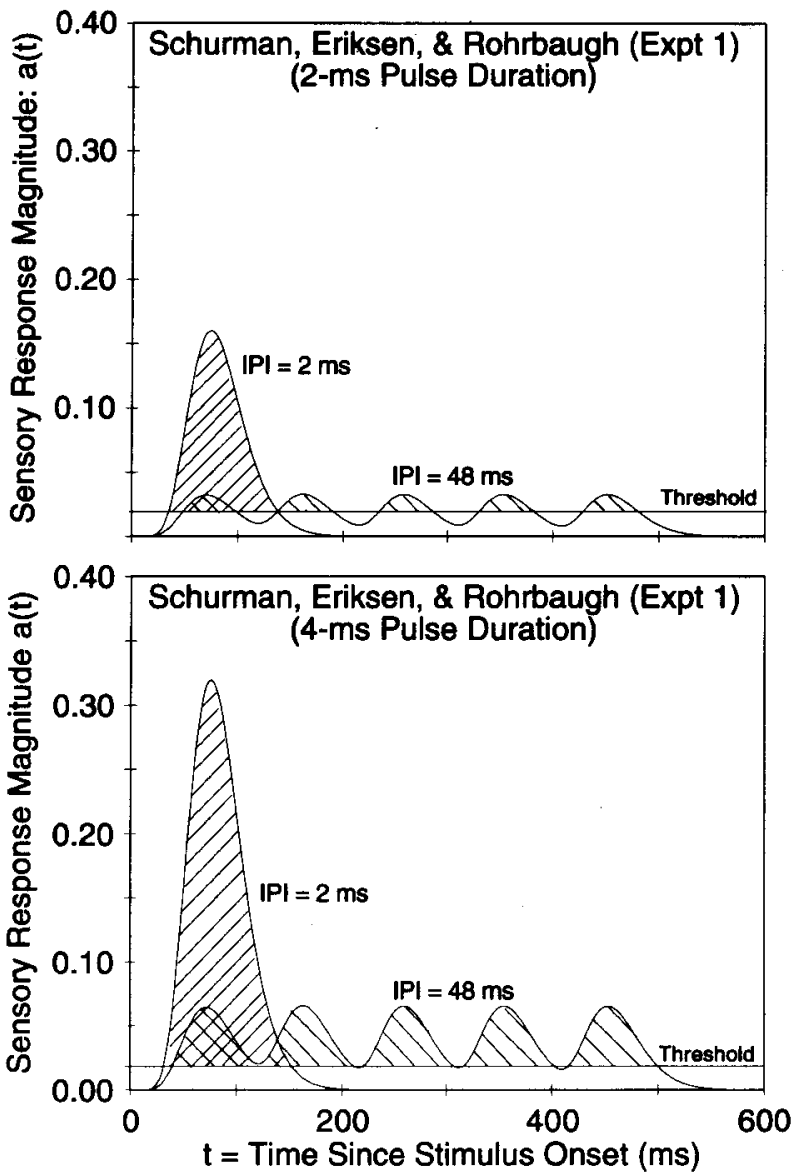

Figure 10. A sketch of how the linear-filter model accounts for data reported by Schurman, Eriksen, and Rohrbaugh (1968). The two panels depict the a $(t)$ functions for trials with 2 -msec pulses (top panel) and with 4-msec pulses (bottom panel). Within each panel, the two curves are for 2 -msec and 48 -msec interpulse intervals.

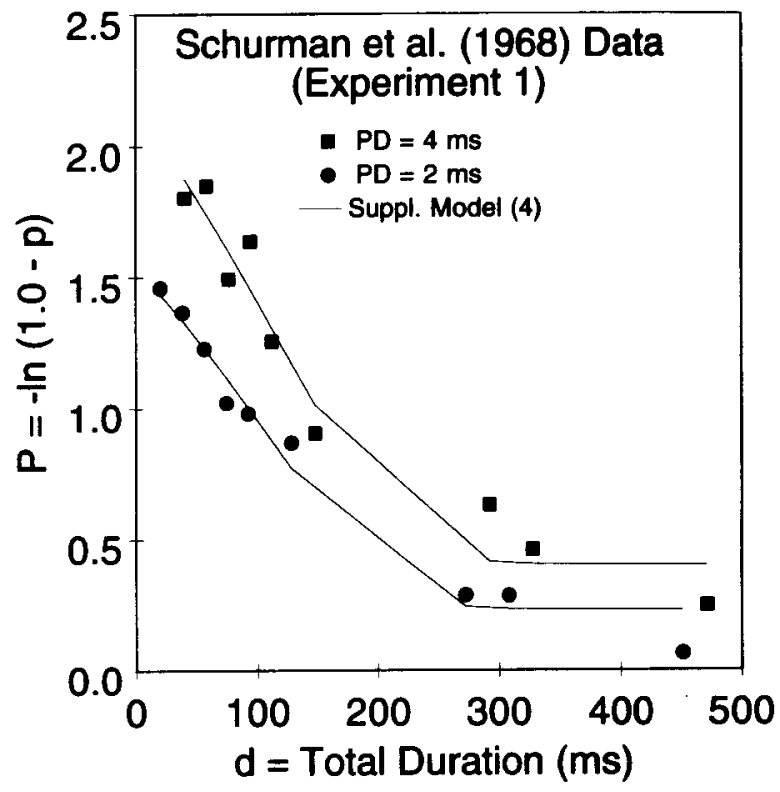

Figure 11. Fit of the supplemented model to the data of Schurman, Eriksen, and Rohrbaugh (1968). Four parameters were required to fit the model to all data points.

\section{Supplemented Model: Quantitative Fits}

The model has four basic parameters: $n$ and $\tau$, the two parameters of the impulse-response function, the sensory threshold, which we designate $a_{\text {thresh }}$, and the proportionality constant between performance-relevant area and $P$, which we designate $1 / c .{ }^{14}$ As shown in Appendix B, the equation that describes performance curves becomes

$$
P=A_{t}(d) / c,
$$

where $A_{t}(d)$ is the magnitude of the performance-relevant area for a $d$-msec stimulus (determined by the parameters $n, \tau$ and $a_{\text {thresh. }}$ ) Note that, as indicated in Figure 9, there is only a single performance-relevant area for an unprimed stimulus, whereas there are two performancerelevant areas-one coming from the prime, and one coming from the primed stimulus-for the primed-stimulus condition.

Basic versus supplemented models. The fits, measured by the root mean square errors (RMSEs), are comparable for the basic and supplemented models. In general, the supplemented model gains an advantage in parsimony over the basic model with more complicated designs. Thus, with an exposure-duration-only design (e.g., a single Experiment 1 observer, as in Figure 3), the basic model requires only three parameters $(c, L$, and $A)$, whereas the supplemented model requires four parameters $(\tau$, threshold, $c$, and $A$; note that $n$ is always set to 9 , not estimated). When an additional, two-level factor is added (e.g., number of targets, as in Figure 2), the basic model requires six parameters (two values each of $c, L$, and $A$ ) and the supplemented model also requires six $(\tau$, threshold, two values of $c$, and two values of $A$ ). When serial position is added as a factor (Figure 4), the basic model requires nine parameters (four values of $c$ and $L$, plus the com- 
mon $A$ ) and the supplemented model requires seven ( $\tau$, threshold, four values of $c$, and the common $A$ ). Finally, for the Experiment 2 data, the basic model requires six parameters (two values of $c$ and $L$, plus the mean primeonly value, plus the common $A$ ) and the supplemented model requires four ( $\tau$, threshold, $c$, and the common $A$ ). The general idea is straightforward: with increasingly complex designs, the supplemented model requires relatively fewer parameters than the basic model does.

To fit the various data sets, we fixed $n$ at 9 , a value often found in the vision literature (see Watson, 1986). We determined the best fits for Shibuya and Bundesen's (1988) data (Figure 2), the Experiment 1 data (Figures 3 and 4), the Experiment 2 data (Figure 7), and Schurman et al.'s (1968) data (Figure 11) by using a least-squares grid search procedure. The best-fitting parameters are shown in Tables 6-10. Each table provides the values of all estimated parameters, along with the RMSEs for both the supplemented model and the basic model. Footnotes to each table provide the number of free parameters upon which each of the RMSEs is based (i.e., the number of data points minus the number of estimated parameters).

The foregoing logic has presumed that the basic model has no means of predicting relations among the slope and intercept values for different serial positions or different priming conditions, for example. This is because the basic model is just that-basic. As we have noted earlier, other investigators have offered other models whose purpose is to predict just such relations.

Supplemented model parameter values. It should be noted that the threshold values are in units of contrast. The rationale underlying this assertion is as follows. As the stimulus remains on indefinitely, $a(t)$ will asymptote at a value equal to stimulus contrast. ${ }^{15}$ Accordingly, the threshold can be interpreted as the maximum stimulus contrast level at which no information would be extracted even if the stimulus were of infinite duration.

Estimated threshold values. For the most part, the threshold values are around $0.005-0.030$. There are some

Table 6

Fit of the Supplemented Random-Sampling ModeI for the 2 Observers in Shibuya and Bundesen (1988)

RMSE*

\begin{tabular}{ccccccc} 
Observer & $\tau$ & $a_{\text {thresh }}$ & $c(T=2)$ & $c(T=4)$ & Supplemented & Basic \\
\hline M.P. & 2.6 & 0.000 & 27.59 & 53.38 & 0.374 & 0.364 \\
H.V. & 2.4 & 0.051 & 23.07 & 73.73 & 0.435 & 0.431 \\
\hline
\end{tabular}

*Twelve free parameters.

Table 7

Fit of the Supplemented Random-Sampling Model for $\mathbf{4}$ Observers in Experiment 1

\begin{tabular}{lccccc}
\hline & & & & \multicolumn{2}{c}{ RMSE } \\
\cline { 5 - 6 } Observer & $\tau$ & $a_{\text {thresh }}$ & $c$ & Supplemented* & Basic $\dagger$ \\
\hline S.S. & 5.2 & 0.031 & 0.215 & 0.036 & 0.041 \\
E.F. & 5.7 & 0.027 & 0.179 & 0.140 & 0.118 \\
T.B. & 6.4 & 0.032 & 0.029 & 0.097 & 0.108 \\
S.T. & 6.2 & 0.119 & 2.11 & 0.041 & 0.046 \\
\hline
\end{tabular}

*Five free parameters. †Four free parameters.
Table 8

Fit of the Supplemented Random-Sampling Model Applied to Four Serial Positions in Experiment 1 (Averaged Over 3 Observers)

\begin{tabular}{cccccccc}
\hline & \multicolumn{4}{c}{$c$} & & & \multicolumn{2}{c}{ RMSE } \\
\cline { 2 - 5 } \cline { 7 - 8 }$a_{\text {thresh }}$ & SP1 & SP2 & SP3 & SP4 & & Supplemented* & Basict \\
\hline 0.026 & 0.308 & 0.296 & 0.532 & 1.085 & & 0.089 & 0.090 \\
\hline
\end{tabular}

*Twenty-five free parameters. $†$ Twenty-three free parameters.

Table 9

Fit of the Supplemented Random-Sampling Model in Experiment 2 (Averaged Over 4 Observers)

\begin{tabular}{ccccc} 
& & & \multicolumn{2}{c}{ RMSE } \\
\cline { 3 - 5 }$\tau$ & $a_{\text {thresh }}$ & $c$ & Supplemented & Basic $\dagger$ \\
\hline 8.7 & 0.0046 & 2.651 & 0.077 & 0.060 \\
\hline
\end{tabular}

*Fourteen free parameters. †Eleven free parameters.

Table 10

Fit of the Supplemented Random-Sampling Model for Schurman, Eriksen, and Rohrbaugh (1968), in Experiment 1 (Averaged Over Observers)

\begin{tabular}{|c|c|c|c|c|c|}
\hline \multirow[b]{2}{*}{$\tau$} & \multicolumn{2}{|c|}{$a_{\text {thresh }}$} & \multicolumn{2}{|c|}{$c$} & \multirow[b]{2}{*}{ RMSE* } \\
\hline & $2 \mathrm{msec}$ & $4 \mathrm{msec}$ & $2 \mathrm{msec}$ & $4 \mathrm{msec}$ & \\
\hline 2.6 & 0.075 & 0.138 & 11.37 & 16.6 & 0.124 \\
\hline
\end{tabular}

*Ten free parameters.

exceptions. First, Observer M.P. in Shibuya and Bundesen's (1988) study had an estimated threshold of 0.00 . Second, Observer S.T. in Experiment 1 had an estimated threshold of 0.119. Recall that S.T. was unpracticed; accordingly, one might expect his threshold value to have been higher than those of the practiced observers. The thresholds were also somewhat higher for the Schurman et al. (1968) data. We are not sure why this was so; it could have been due to the observers' practice levels or to the particular nature of the luminances that were used.

Estimated $\mathrm{c}$ values and processing efficiency. The estimated $c$ values are of some interest, because they provide measures of the overall processing efficiency for a given condition. In Appendix C, we provide details of how such an efficiency measure can be derived from our theory, along with the observed values both from the present experiments and from Shibuya and Bundesen's (1988) experiment.

\section{Bloch's Law}

There is a well-known tradeoff between stimulus intensity and stimulus duration for short-duration stimuli (e.g., Hood \& Grover, 1974; Kahneman, 1968; Kahneman \& Norman, 1964; Kaswan \& Young, 1963; Zacks, 1970; see Watson, 1986, and Wasserman \& Kong, 1979, plus associated commentaries, for reviews).

Equation 8 (above), which generates the $a(t)$ functions, implies a tradeoff between contrast and duration. Consider the situation in which there is no masking and the sensory threshold is zero. Under such conditions, the performancerelevant area is equal to the product of duration and contrast; accordingly, a perfect Bloch's law tradeoff is predicted. When there is masking, or an above-zero threshold, 
the tradeoff is not perfect, although Loftus and Ruthruff (in press) showed that in a no-mask experiment, with threshold estimated to be above zero, a form of Bloch's law continues to occur for contrasts ranging from 0.033 to approximately 0.21 . It is clear from the present experiments that such a tradeoff could not be extended up to a contrast level of 1.0: the implied sampling rates (Appendix $C$, Table 11, bottom row) are simply too large.

Earlier, we noted a difference between the magnitudes of liftoff values found by Shibuya and Bundesen (1988; on the order of 10-30 msec) and in the present experiments (on the order of 50-100 msec) and asserted that the difference could well be due to the higher contrast values used by Shibuya and Bundesen. The supplemented model puts us in a position to explain this assertion as follows. With higher contrast, the $a(t)$ curves are higher; accordingly, a shorter exposure duration is required for a given $a(t)$ curve to exceed threshold (see Loftus and Ruthruff, in press, for a detailed explanation).

\section{Linear Performance Curves}

Earlier in this article, we noted that the linear functions embodied in Equation 7 describe extant performance curves quite well. When the sensory-response functions of the sort depicted in Figure 8 are used as the basis of the information-acquisition process, Equation 10 rather than Equation 7 generates the performance curves. Although Equation 10 does not generate perfectly linear performance curves, it does generate, as is evident in Figures $1,3,4$, and 7 , approximately linear curves.

\section{Deficiencies}

There are two characteristics of our Experiment 2 data that are not completely in compliance with the predictions of the supplemented model. We describe them in turn.

Nonparallel primed and unprimed performance curves. We noted earlier that, in Experiment 2, the average primed and unprimed performance-curves slopes were the same (see Table 5). As we also noted, however, 2 of the 4 observers failed to conform to this pattern: for $\mathrm{Ob}-$ server G.L., the parameter $c$ was larger in the primed than in the unprimed condition (155 vs. $132 \mathrm{msec}$, a $17 \%$ difference), whereas the opposite was true for Observer T.J.B. (62 vs. $77 \mathrm{msec}$, a $24 \%$ difference).

Within the supplemented model's context, such a discrepancy could arise for either or both of two reasons. First, as indicated in Figure 5C, the sampling rate could depend on whether a stimulus has or has not been preceded by a prime. Second, the sensory threshold could depend on whether a stimulus has or has not been preceded by a prime. There is no obvious reason to prefer one possibility over the other, and the individual data are not sufficiently powerful to disambiguate them empirically.

Nonrandom sampling. As can be seen in Figure 7, there were slight but systematic deviations between the data points and the supplemented model's prediction. These deviations arise because the obtained primed and unprimed performance curves are slightly closer together (i.e., the prime's worth is slightly smaller) than the model predicts. As discussed earlier, a plausible explanation for this effect is that it stems from a small degree of nonrandom sampling in the primed-stimulus condition. We have already noted one possible reason for this: when the primed stimulus is presented, some of the features already sampled from the prime may be systematically resampled from the primed stimulus. As an example of how this might happen, suppose that stimulus onset biased the observer to attend to the leftmost digit of the stimulus array. Then the leftmost digit would be systematically resampled during primed-stimulus presentation even if it had already been acquired from the prime. In order to predict these small deviations, the model would have to incorporate additional assumptions about the nature of systematic resampling. Such assumptions are beyond the scope of the present treatment.

\section{CONCLUSIONS}

We have developed a model that represents a marriage of two traditions: the basic random-sampling model from the memory and attention literature, and the linear-filter model from the vision and sensation literature. The resulting supplemented random-sampling model anchors the basic random-sampling model in a closer approximation of sensory reality. It also subsumes the basic model, in that it generates (approximately) the same linear performance curves and also accounts for two facets of the data-the processing delay (liftoff) and the prime's worth paradox - that are not predicted by the basic model.

We wish to reiterate that there is an important domain about which our model is silent-that involving spatial and temporal distribution of attention across different stimulus items (e.g., different characters within a character array). This domain has been investigated assiduously by others (e.g., Rumelhart, 1970; Shibuya \& Bundesen, 1988; Townsend, 1981; see Bundesen, 1990, for a review). However, it was not our intention to address these issues; our principle concern was to supplement the basic random-sampling model with a more realistic sensory basis. The supplement that we have described could easily be incorporated into the attentional models offered by other investigators.

\section{REFERENCES}

Averbach, E., \& Sperling, G. (1961). Short-term storage in vision. In C. Cherry (Ed.), Symposium on information theory (pp. 196-211). London: Butterworth.

Bundesen, C. (1990). A theory of visual attention. Psychological Review, 97, 523-547.

BusEy, T. A., \& LoFTUS, G. R. (in press). Linear and nonlinear components of visual information acquisition. Psychological Review.

Cornsweet, T. N. (1971). Visual perception. New York: Academic Press

DE LANGe, H. (1952). Experiments of flicker and some calculations on an electrical analogue of the foveal systems. Physica, 18, 935-950.

ERIKSEN, C. W. (1980). The use of a visual mask may seriously confound your experiment. Perception \& Psychophysics, 28, 89-92. 
HoOD, D. C., \& Grover, B. G. (1974). Temporal summation of light by a vertebrate visual receptor. Science, 184, 1003-1005.

IvEs, H. E. (1922). A theory of intermittent vision. Journal of the Optical Society of America, 6, 343-361.

KAHNEMAN, D. (1968). Method, findings, and theory in studies of visual masking. Psychological Bulletin, 70, 404-425.

KAHNeman, D., \& NoRMAN, D. (1964). The time-intensity relation in visual perception as a function of the observer's task. Journal of $E x$ perimental Psychology, 65, 215-220.

Kaswan, J., \& Young, S. (1963). Stimulus exposure time, brightness, and spatial factors as determinants of visual perception. Journal of Experimental Psychology, 65, 113-123.

Kelly, D. H. (1961). Visual responses to time-dependent stimuli: I. Amplitude sensitivity measurements. Journal of the Optical Society of America, 51, 422-429.

Kowler, E., \& Sperling, G. (1980). Transient stimulation does not aid visual search: Implications for the role of saccades. Perception \& Psychophysics, 27, 1-10.

Loftus, G. R., Duncan, J., \& Gehrig, P. (1992). On the time course of perceptual information that results from a brief visual presentation. Journal of Experimental Psychology: Human Perception \& Performance, 18, 530-549.

Lofrus, G. R., Johnson, C. A., \& Shimamura, A. P. (1985). How much is an icon worth? Joumal of Experimental Psychology: Human Perception \& Performance, 11, 1-13.

Lortus, G. R., \& Kallman, H. J. (1979). Encoding and use of detail information in picture recognition. Journal of Experimental Psychology: Human Learning \& Memory, 5, 197-211.

LofTUs, G. R., \& RUTHRUFF, E. (in press). A linear-filter theory of visual information acquisition with special application to intensityduration tradeoffs. Journal of Experimental Psychology: Human Perception \& Performance.

Massaro, D. W. (1970). Perceptual processes and forgetting in memory tasks. Psychological Review, 77, 557-567.

NeIMARK, E. D., \& EsTes, W. K. (1967). Stimulus sampling theory. San Francisco: Holden Day.

Reinitz, M. T., Wright, E., \& Loftus, G. R. (1989). The effects of semantic priming on visual encoding of pictures. Journal of Experimental Psychology: General, 118, 280-297.

RumelharT, D. E. (1970). A multicomponent theory of the perception of briefly exposed visual displays. Journal of Mathematical Psychology, 7, 191-218.

Rundus, D. J. (1971). Analysis of rehearsal processes in free recall. Journal of Experimental Psychology, 89, 63-77.

Schurman, D. L., Eriksen, C. W., \& Rohrbaugh, J. (1968). Masking phenomena and time-intensity reciprocity for form. Journal of Experimental Psychology, 78, 310-317.

SHibUYA, H., \& BUNDESEN, C. (1988). Visual selection from multielement displays: Measuring and modeling effects of exposure duration. Journal of Experimental Psychology: Human Perception \& Performance, 14, 591-600.

SPERLING, G. (1960). The information available in brief visual presentations. Psychological Monographs, 74, 1-29.

SPERLING, G. (1964a). Linear theory and the psychophysics of flicker. Documenta Ophthalmologica, 18, 3-15.

SPERLING, G. (1964b). What visual masking can tell us about temporal factors in perception. In Proceedings of the Seventeenth International Congress of Psychology (pp. 199-200). Amsterdam: North-Holland.

STODDARD, P. K., \& LofTUS, G. R. (1988). An IBM XT-compatible, computer-based, slide-projector laboratory. Behavior Research Methods, Instruments, \& Computers, 20, 541-551.

Townsend, J. T. (1981). Some characteristics of visual whole report behavior. Acta Psychologica, 47, 149-173.

Wasserman, G. S., \& KonG, K. L. (1979). Absolute timing of mental activities. Behavioral \& Brain Sciences, 2, 243-255.

Watson, A. B. (1986). Temporal sensitivity. In K. R. Boff, L. Kaufman, \& J. P. Thomas (Eds.), Handbook of perception and human performance (Vol. 1, pp. 6-1 to 6-41). New York: Wiley.

WOLFORD, G. (1975). A perturbation model for letter identification. Psychological Review, 82, 184-199.

Wolford, G., \& Chambers, L. (1983). Lateral masking as a function of spacing. Perception \& Psychophysics, 33, 129-138.
ZACxs, J. L. (1970). Temporal summation phenomena at threshold: Their relation to visual mechanisms. Science, 170, 197-199.

\section{NOTES}

1. To check this assumption, we carried out a control procedure in which we displayed the stimuli concurrently with the mask. Even when this stimulus-mask conjunction was displayed indefinitely, no stimulus digits could be seen; recall performance was at chance.

2. This development of the random-sampling model begs the question of what, exactly, is randomly sampled. The mathematics are consistent with several possibilities. One is that each target digit is made up of a collection of features (e.g., lines, angles, etc.) that are sampled (as in Rumelhart's, 1970, multicomponent theory), in which case each digit would be recalled with a probability equal to the proportion of that digit's total acquired features. Another possibility is that the basic unit is the target digit, which is sampled according to a Poisson process as in Shibuya and Bundesen's (1988) race model or Townsend's (1981) bounded performance model. For our purposes, it is not relevant which of these possibilities is supposed.

3. The proportion correct, $p$, is corrected for the chance value of .10 .

4. More precisely, this control procedure was as follows. The digits appeared and remained on until the observer had read them, at which time he/she pressed a key. The digits then disappeared, and the observer entered them into the response box. Performance (correct digits in correct position) was over $95 \%$. The observers were required to explain the infrequent errors: all occurred as a result of inadvertently pressing the wrong key on the keypad.

5. We thank Claus Bundesen for providing these data.

6. The 80 stimulus slides were fixed in the 80 slots of a carousel tray. We wanted to preclude the observers' ability to memorize and make use of sequential, slide-to-slide information (e.g., we did not want the bottom row " 7184 " to always follow the bottom row " 0072 "). We accomplished this goal as follows. On each block, the 72 experimental stimuli were randomly divided into two 36-stimulus groups (Groups A and $B$ ). The carousel circled twice within each block: on Pass 1, all Group A stimuli were shown, and on Pass 2, all Group B stimuli were shown. This scheme ensured that stimulus ordering differed unpredictably from one block to the next.

7. Actually, because the curves are linear, the primed and unprimed curves are vertically as well as horizontally parallel. We emphasize horizontal parallelness, because any nonlinear transform on the dependent variable (e.g., expressing performance in terms of $p$ or $d^{\prime}$ rather than $P$ ) will preserve the horizontal, but not the vertical parallelness.

8. This prediction, like that of Figure 3B, assumes that whatever causes the processing delay (the nonzero liftoff) will affect the primed and unprimed conditions similarly.

9. The impulse-response function is the system's response to an instantaneous stimulus impulse (a mathematical fiction defined to be a stimulus of infinitesimal duration, infinite contrast, and unit area).

10. It is also apparent in Figure 8 that, according to the model, a mask is not necessary to produce an above-zero liftoff, although liftoff would be smaller without a mask. In particular, it would be the largest duration such that the maximum of the ensuing sensory-response function just reached threshold. Busey and Loftus (in press) and Loftus and Ruthruff (in press) confirm this prediction.

11. Note, however, that even under these circumstances there would still be some information obtained from the primed stimulus, because the peak magnitude of the 100 -msec primed stimulus exceeds the peak magnitude of the 50-msec prime.

12. If the primed stimulus were not masked, this maximum prime's worth would be $50 \mathrm{msec}$, the prime's physical duration. With a mask, the maximum prime's worth is somewhat more than $50 \mathrm{msec}$; how much more depends on the specific parameters of the $a(t)$ function.

13. For simplicity's sake, 5 rather than 10 pulses are shown in Figure 10 . The argument would be the same no matter how many pulses were used.

14. The proportionality constant is expressed this way because it allows the parameter $c$, expressed in milliseconds, to be related to the $c$ in the original linear function described by Equations 6 and 7. This relation is discussed in more detail below. 
15. The reason for this can be seen in Equation 8 , above. The total area under $g(t)$ is 1.0 . Thus, as $d$ becomes indefinitely large, $G(t)$ asymptotes at 1.0 , and $a(t)$ thus asymptotes at $\phi$, the contrast level.

\section{APPENDIX A}

\section{Derivation of Text Equation 2, and Related Matters}

Let the raw sampling rate be $1 / c$; that is, features are always sampled at a rate of $1 / c$ per unit time. Whenever a feature is sampled, it is transferred to short-term memory. At time $t$ following stimulus onset, some proportion, $I(t)$, of the features has been sampled; these features are in short-term memory.

The remaining proportion, $[1.0-I(t)]$, are new featuresfeatures that have never been sampled. Accordingly, at time $t$ following stimulus onset, the proportion of the $(1 / c)$ sampled features that are new is $[1.0-I(t)]$, which means that the rate of sampling new features, $r(t)$, is

$$
r(t)=\frac{1.0-I(t)}{c}
$$

Because $r(t)$ is the derivative of new features, $I(t)$ with respect to time, Equation Al may be rewritten as

$$
\frac{d[I(t)]}{d t}=\frac{1.0-I(t)}{c} .
$$

With terms rearranged,

$$
\frac{d[I(t)]}{1.0-I(t)}=\frac{d t}{c} .
$$

Integrating both sides of Equation A3,

$$
-\ln [1.0-I(t)]=\frac{t}{c}+k
$$

where $k$ is the constant of integration. When $t=0, I(t)=0$, which implies that $k=0$. Moreover, following the mask that occurs at stimulus offset (time $d$ ), no more features are acquired. Thus, the total of features acquired from the stimulus is obtained by setting $t$ to $d$. Setting $t$ to $d$, setting $k$ to 0 , and exponentiating Equation $\mathrm{A} 4$ yields,

$$
1.0-I(t)=e^{-d / c},
$$

or, with terms rearranged,

$$
I(t)=1.0-e^{-d / c},
$$

which is text Equation 2.

\section{APPENDIX B \\ Derivation of the Performance-Curve Function Generated by the Supplemented Model}

We carry out this derivation in two parts. First, for the record, we show that, under weak assumptions, performance is monotonically related to the performance-relevant area. We then show that under assumptions of the random-sampling model, our performance measure, $P$, is proportional to this area.

Let a function $a_{t}(t)$ be the magnitude by which $a(t)$ exceeds threshold. Thus, $a_{t}(t)$ may be defined as

$$
a_{t}(t)=\begin{array}{ll}
0 & \text { for } a(t) \leq a_{\text {thresh }} \\
a(t)-a_{\text {thresh }} & \text { for } a(t)>a_{\text {thresh }}
\end{array}
$$

where $a_{\text {thresh }}$ is the magnitude of the sensory threshold.
Also, let $r(t)$, the feature-acquisition rate, be the product of $a_{t}(t)$ and $h[I(t)]$, where $h[I(t)]$ is a monotonically decreasing, positive function of $I(t)$, the proportion of acquired features at time $t$. Let $h[I(t)]$ be further constrained in such a way that it approaches 0 as $I(t)$ approaches 1.0.

Four remarks about this form of $r(t)$ are in order. First, $I(t)$ is automatically constrained to be less than 1.0. Second, $r(t)$ is zero whenever $a_{t}(t)=0$. Third, this form of $r(t)$ is a generalization of that described by Equation Al (above in Appen$\operatorname{dix} \mathrm{A})$, in that $h[I(t)]$ is not constrained to be $[1.0-I(t)] / c$, and $a_{t}(t)$ is not constrained to be 1.0. Fourth, the performance curves generated by this form of $r(t)$ are not perfectly exponential; however, they are so close as to be experimentally indistinguishable from exponential curves.

The feature-acquisition rate, $r(t)$, is, by definition, the derivative of acquired features, $I(t)$, with respect to time. Thus,

$$
r(t)=\frac{d[I(t)]}{d t}=a_{t}(t) h[I(t)]
$$

or

$$
\frac{d[I(t)]}{h[I(t)]}=a_{t}(t) d t
$$

Integrating both sides of Equation B1,

$$
H[I(t)]=A_{t}(t)+k,
$$

where $A_{t}(t)$ is the integral of $a_{t}(t), H[I(t)]$ is the integral of $\{1 / h[I(t)]\}$, and $k$ is the constant of integration. When $t=0$, $A_{t}(t)=0$ and $I(t)=0$; hence, $k=H(0)$. Therefore,

$$
H[I(t)]=A_{t}(t)+H(0) .
$$

Because $H$ is an integral, it is monotonically increasing and has an inverse, $H^{-1}$, which is also monotonic. From Equation B2,

$$
I(t)=H^{-1}\left[A_{t}(t)+H(0)\right] .
$$

Because the mask does not allow any acquisition of information beyond time $t=d$, which is the stimulus duration, the total acquired information is obtained by setting $t$ to $d$ :

$$
I(d)=H^{-1}\left[A_{t}(d)+H(0)\right] .
$$

Therefore $I(d)$ is a monotonic function of $A_{t}(d)$, which, in turn, is by definition the performance-relevant area. If $p$ is assumed to be a monotonic function of $I(d)$, the total acquired information, then $p$ is also a monotonic function of the performancerelevant area.

Suppose that we now assume feature sampling to occur at the rate of $a_{t}(t) / c$. By the logic provided in Appendix $\mathrm{A}, h[I(t)]=$ $(1.0-I) / \mathrm{c}$. Therefore,

$H[I(t)]=\int_{0}^{I(t)} \frac{c d t}{1.0-I(t)}=-c \ln [1.0-I(t)]-c \ln (1.0-0)$,

or

$$
H[I(t)]=-c \ln [1.0-I(t)] .
$$

Note also that $H^{-1}(x)=\left(1.0-e^{-x / c}\right)$, and that $H(0)=0$. Thus, by Equation B3,

$$
I(d)=1.0-e^{-A_{i}(d) / c} .
$$

Assuming performance, $p$, to equal $I(d)$, then

$$
1.0-p=e^{-A_{t}(d) / c} \text {. }
$$


Table C1

Calculation of Sampling Rates (Items/Second) From the Basic Model and From the Supplemented Model

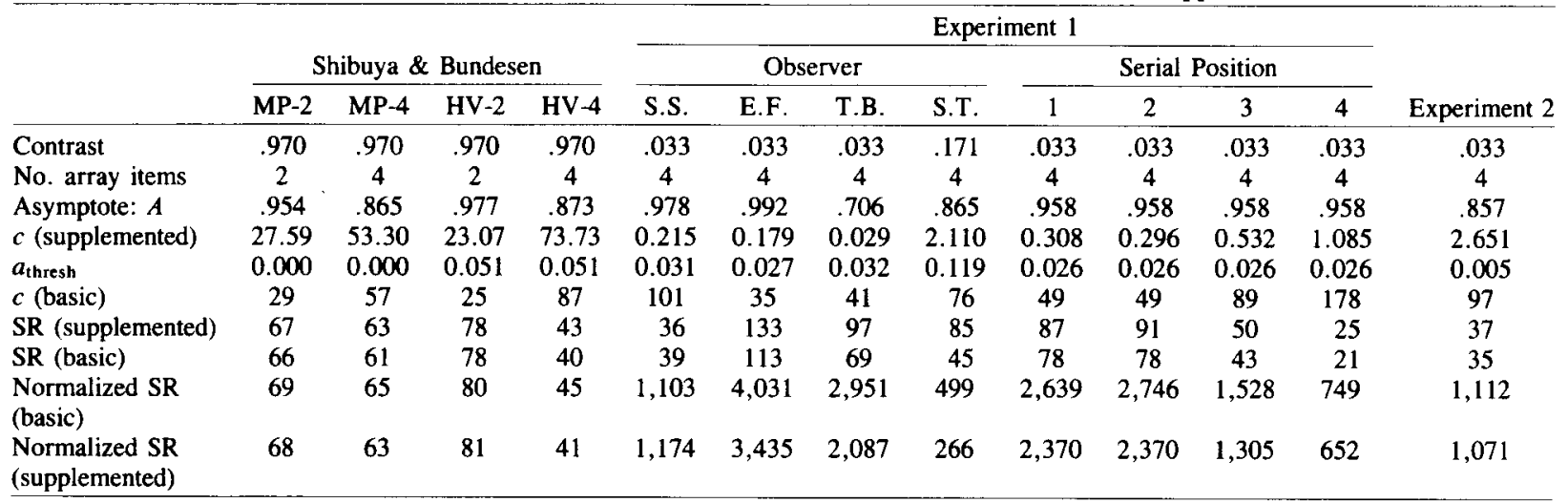

Note-SR, sampling rate, in items/second.

Taking the logarithm of both sides of Equation B4,

$$
-\ln (1.0-p)=A_{t}(d) / c \text {. }
$$

Because $-\ln (1.0-p)=P$, Equation B5 is text Equation 10 . This completes the proof.

\section{APPENDIX C \\ Details of Estimating Encoding Efficiency Under Various Circumstances}

Table $\mathrm{Cl}$ provides some derived information regarding encoding efficiency, for both the supplemented model and the basic model. Table $\mathrm{C} 1$ is organized as follows. The columns represent the 13 data sets that we have previously considered in this article. For each data set, the top two rows provide independent variables: contrast and number of array items. The next four rows provide the asymptote, two parameters $-c$ and threshold-for the supplemented model, and the parameter $c$ for the basic model.

This information enables us to provide estimates of processing efficiency, measured in acquired items/second, from the two models. These estimates are provided in the next two rows and have been obtained as follows. For the basic model, the raw sampling rate is $1 / c$ (see Appendix A). To arrive at features/ second one multiplies this value by 1,000 (to change from milliseconds to seconds), then multiplies by the number of array items (to change from percent features to items), and finally multiplies by the asymptote to arrive at the raw sampling rate in items/second.

The efficiency calculation is somewhat more complex for the supplemented model. Consider the $a(t)$ functions shown in Figure 8 . According to the model, sampling rate is proportional to $a_{t}(t)$ at any given time $t$. To compute efficiency, we have chosen to consider the maximum sampling rate-that is, the rate that occurs when the stimulus has been on indefinitely. We have noted that, under such conditions, $a(t)$ asymptotes at the contrast value, $\phi$, at which point $a_{t}(t)$ is equal to the contrast value minus the threshold value. We refer to this asymptotic $a(t)$ value as $a_{\max }$.

By the random-sampling assumptions, the raw sampling rate continues to be equal to $1 / c$ within the supplemented-model's context. However, this rate must now be multiplied by $a_{\max }$ to obtain the maximum raw sampling rate, in percent/millisecond. The logic then proceeds as in the basic model (multiplying by $1,000 \times$ number of array items $\times A$ ) to arrive at estimated raw sampling rate in items/second.

The final two rows of Table $\mathrm{C} 1$ provide the normalized sampling rate for the two models. This figure is computed supposing a contrast of 1.0, under the assumption that sampling rate is indeed proportional to contrast. Accordingly, for each model, the normalized sampling rate is simply the ordinary sampling rate divided by contrast.

The estimated sampling rates in Table $\mathrm{Cl}$ allow conclusions of various kinds. First, the ordinary (nonnormalized) sampling rates estimated by both models are in the 50-100 items/second range, which is comparable to those estimated by others. Second, the rates estimated from the supplemented model are somewhat greater than those estimated by the basic model. The reason for this is that the basic model assumes (implicitly) that when the sensory response appears, it is at its maximum (of 1.0); therefore, the raw sampling rate $(1 / c)$ is, according to the basic model, the only (and thus highest) value that this rate can assume. According to the supplemented model, however, the sensoryresponse function grows over time, and therefore the rates that occur within an experiment-particularly with these rather shortduration stimuli-are lower than the theoretical maximum. Third, the normalized sampling rates, while reasonable for Shibuya and Bundesen's (1988) data, are unrealistically high for the data from Experiments 1 and 2. This indicates that one cannot simply extrapolate linearly from the contrast that we used $(0.033)$ to make conclusions about the nature of processing at arbitrarily high contrast levels.

The scanning rates according to the supplemented model can also be computed for Schurman et al.'s (1968) data, although they are maximum rates because no asymptote can be estimated, and the asymptote is set at 1.0. Nonetheless, they fall within the normal range: they are 81 and 52 items/msec for the 2- and 4-msec stimuli. We do not know why there is a difference between the two stimulus durations. Perhaps because presentation was blocked by stimulus duration, different strategies emerged for the two durations.

(Manuscript received July 13, 1992; revision accepted for publication March 26, 1993.) 